Noname manuscript No.

(will be inserted by the editor)

\title{
Self-generated limit cycle tracking of the underactuated inertia wheel inverted pendulum under IDA-PBC
}

\author{
Hassène Gritli · Nahla Khraief • Ahmed Chemori · Safya \\ Belghith
}

Received: date / Accepted: date

\begin{abstract}
This paper deals with the tracking approach of a self-generated stable limit cycle for an underactuated mechanical system: The Inertia Wheel Inverted Pendulum (IWIP). Such system is subject to unilateral constraints limiting its swing motion. It is known that an Interconnection and Damping AssignmentPassivity Based Control (IDA-PBC) can be employed to control such pendulum to its upright position. In this work, we briefly show first that the IWIP can generate a stable period-1 limit cycle through a Hopf bifurcation by varying some gain parameter of the IDA-PBC. Thus, such self-generated limit cycle is used as a reference trajectory, which is chosen to be tracked by the IWIP. To achieve the tracking problem, a supplementary control input is added. Such tracking problem is reformulated as an asymptotic stabilization of the tracking error. Our fundamental approach hinges mainly on the use of the S-procedure to introduce the unilateral constraints, and the Schur complement and the matrix inversion lemma to transform Bilinear Matrix Inequalities (BMI) into Linear Matrix Inequalities (LMI). Several simulations have been presented to corroborate the mathematical results and to show the efficiency of the proposed tracking scheme of the self-generated stable limit cycle of the controlled IWIP, even if it is subject to external disturbances, or in the presence of uncertainties in the friction parameters.
\end{abstract}

Keywords Inertia wheel inverted pendulum · Hopf-bifurcation · Limit cycle • Unilateral constraint · S-procedure · BMI · LMI

\section{Introduction}

Equilibrium points and limit cycles are known to be common solutions for almost all continuous-time nonlinear dynamical systems. Limit cycles model dynamical systems that exhibit self-sustained oscillations for some set of parameters. Thus, a self-excited system exhibits the property to generate steady state oscillations (limit cycles) even in the absence of external periodic forcing [1-3]. Some examples of dynamical systems that exhibit a self-excited oscillation are beating of a heart, rhythms in body temperature, hormone secretion, chemical reactions, vibrations in bridges and airplane wings, inverted pendulums, the Van der Pol oscillator, the Duffing oscillator, biped robots, etc. [4-7] (see also [8, 9] for further examples). In general, selfoscillators give a regular output robustly: they approach the same limit cycle regardless of initial conditions and transient perturbations [2]. In addition, limit cycles are generated through bifurcations, among which the most popular and important one is the Hopf bifurcation [1, 4, 6, 10], which occurs for autonomous

\section{H. Gritli*}

Institut Supérieur des Technologies de l'Information et de la Communication,

Université de Carthage, 1164 Borj Cedria, Tunis, Tunisia

^E-mail: grhass@yahoo.fr

H. Gritli · N. Khraief · S. Belghith

Laboratoire Robotique, Informatique et Systèmes Complexes (RISC-LR16ES07),

Ecole Nationale d'Ingénieurs de Tunis, Université de Tunis El-Manar,

BP. 37, Le Belvédère, 1002 Tunis, Tunisia

A. Chemori

LIRMM, University of Montpellier 2, CNRS, 161 rue Ada, 34392 Montpellier, France 
dynamical systems. Such local bifurcation gives rise to the birth of a limit cycle via a stable equilibrium point. This limit cycle can be either stable or unstable. In the former case, the Hopf bifurcation is supercritical. However, in the latter case, the Hopf bifurcation is sub-critical $[4,7,10]$.

Recently, the interest in the control problem of underactuated mechanical systems has increased due to their complexity and applications. Several kinds of underactuated mechanical systems exist in literature such as the double inverted pendulum, the rotational inverted pendulum, the Furuta pendulum, the cartpendulum system, the wheeled inverted pendulum, the flywheel inverted pendulum, among others [11-18]. In general, inverted pendulums are very suitable to illustrate many ideas in automatic control of nonlinear systems. A special underactuated mechanical system is the Inertia Wheel Inverted Pendulum (IWIP), which has two degrees of freedom with only one actuator $[19,20]$. Such underactuated mechanical system has attracted the attention of several researchers in robotics and control system engineering where several control strategies have been designed (see for example [11-17] and references therein). The most recognized works are those based on the energy point-of-view. The general control strategy is to swing the underactuated mechanical system, such as the Acrobot, the Pendubot, the IWIP, the Tora system, the Furuta pendulum, in order to bring/stabilize it to the desired (upright) position.

\subsection{Literature review}

The control objective of the IWIP is to stabilize it at the upright position, namely around the unstable equilibrium point. Many works related to this topic have been proposed. The passivity-based control with saturated control input based on the feedback linearization was introduced in [19, 20]. Olfati-Saber [21, 22] employed the standard backstepping procedure. Fantoni and Lozano [15] introduced the total energy stored in the system in order to design a nonlinear swinging-up control strategy. Ramamoorthy and Kuipers [23] developed an approach that uses switching between multiple controllers to robustly stabilize the upright equilibrium. The same problem was solved in [24] with a single output feedback controller, which was designed using a passivity-based method. Moreover, Ortega et al. [25] used the Interconnection and Damping Assignment-Passivity Based Control (IDA-PBC) (see [26] for a survey) for the asymptotic stabilization of the IWIP around its upward position. They formulated the designed controller for Port-Controlled Hamiltonian models [27]. In [28, 29], the undesirable effects of the damping force was taken into account in the control strategy. Qaiser et al. [30] presented a novel nonlinear controller design by fusing the dynamic surface control and the control Lyapunov function method. In addition, Touati and Chemori [31] proposed a generalized predictive control scheme. They proved via experimentation the robustness of such controller against external disturbances and uncertainties in the inertia parameters of the IWIP. Moreover, Olivares and Albertos $[32,33]$ used a simple PID controller and an observer-based state feedback controller by linearizing the dynamics around the unstable equilibrium point. Recently, Khraief et al. [34] employed the IDA-PBC for the asymptotic stabilization of the underactuated IWIP around its unstable upward position in the presence of external disturbances. Some sufficient stability conditions on matched and unmatched disturbances were also presented. Moreover, in [35], Khraief et al. enhanced the IDA-PBC by combining it with an adaptive control technique to estimate controller gains. Ryalat and Laila [36] used a simplified IDA-PBC by considering friction parameters. They estimated the friction parameters in order to calculate the friction compensation term. Some other works were realized on the control of inverted pendulums [17, 37-40].

Another interesting and challenging task with the IWIP is the stabilization of periodic motions. Authors in [41-46] developed a (quasi-continuous) high(second)-order sliding mode control to solve the tracking control problem for the IWIP. These authors developed a reference model (trajectory) based on a two-relay controller, which was introduced to produce oscillations where the desired amplitude and frequency were reached by choosing the control gains properly. The two-relay controller consists of two relays switched by the feedback received from a linear or nonlinear system, and represents a new approach to the self-generation of periodic motions in underactuated mechanical systems and hence to design a (robust) tracking controller [45]. The design procedures for the two-relay control and hence the periodic oscillations developed by Aguilar and his co-workers were based on three different methodologies, namely the describing-function method, the Poincaré maps, and the locus-of-a perturbed-relay-system method. Authors in [44] considered the viscous friction affecting the active joint. Another approach for stabilizing periodic motions around the upper equilibrium of the IWIP was presented by [47]. Furthermore, a constructive method introduced in [48-50] was applied for the generation of periodic motions in underactuated nonlinear systems through virtual holonomic constraints and for orbital stabilization via a partial feedback transformation. Gruber and Hofbaur [51] employed the same method for planning periodic motions and designing a stabilizing controller based on a thorough analysis of the dynamics transversal to the resulting limit cycle. In addition, Andary et al. [52] proposed a control approach dedicated to stable limit cycle generation for the IWIP. The proposed 
approach was based on partial nonlinear feedback linearization and dynamic control for optimal periodic reference trajectories tracking. The feedback controller presented in [52] is enhanced in [53, 54] to handle constant disturbances in limit cycle tracking by using online iterative estimation of an equivalent disturbance which is easily compensated by adding the estimated value to the output of the system. Moreover, ZayaneAissa et al. [55] introduced a high order sliding mode structure to solve the problem of trajectory tracking problem of the IWIP in presence of unknown input perturbation. Aguilar-Ibãnez et al. [56] used the state feedback controller to ensure the tracking of a limit cycle characterized in terms of the feedback-linearizable single-input affine nonlinear dynamical systems and, as an application, of the IWIP.

Some analysis of the IWIP under control have been considered in literature using bifurcation theory. Alonso et al. [57] employed the bifurcation theory to classify different dynamical behaviors arising in the IWIP subject to bounded continuous-state-feedback control law. To limit the maximum amplitude of the control action, the control law is subject to a smooth saturation function, which was introduced first in [58] in order to stabilize the IWIP at the inverted position. Alonso et al. [57] showed that the global dynamics of the IWIP changes from a stable equilibrium point to a stable limit cycle via a Hopf bifurcation as certain control gains change. A similar work was realized on the Furuta pendulum by analyzing the Hopf bifurcation [59]. Recently, Nikolov and Nedev [60] analyzed bifurcation and dynamic behavior of the IWP with bounded control by means of the theory of Poincaré-Andronov-Hopf.

Moreover, in the literature, there are several design methods of the feedback controller using the Linear Matrix Inequality (LMI henceforth) approach [61], such as the state feedback control, the static output feedback control, the dynamic output feedback control, the observer-based feedback control, among others (see for example [62] and references therein). However, most control synthesis problems cannot be written in LMI setting, while in terms of a more general form known as a Bilinear Matrix Inequality (BMI). Computations over BMI constraints are known as NP-hard and difficult to obtain a solution. There are several approaches and different relaxed synthesis conditions proposed for conducting the BMIs into LMIs. Generally, all these approaches use for example the Schur complement Lemma, the Finsler Lemma, the projection Lemma, the Young relation, among others [61]. A brief review on the state feedback control design using LMI methods for continuous-time linear systems was presented in [63]. Some extensions and numerical comparisons between several state feedback stabilization conditions were also provided. Furthermore, a new extended LMI characterization for the state feedback control of continuous-time linear systems with uncertainty was presented in [64].

\subsection{Motivations}

The motivations of studying and controlling the underactuated IWIP mainly stem from five facts:

1. In spite of various efforts on the stabilization of the IWIP, a considerable attention is still required for the generation of stable periodic oscillation for the IWIP and synthesis of a feedback control law to track it. In this present paper, we use the theory of bifurcations to generate a stable one-periodic limit cycle by means of the Hopf bifurcation by varying some control parameter.

2. According to [34, 35, 52-54], the motion of the IWIP is constrained. Indeed, the planar swing motion of the pendulum body is subject to unilateral contacts (constraints) [65], for which its angular position reaches a certain maximum/minimum value at standstill. Olivares and Albertos [33] worked with a physical device having such constraints. To the best of authors' knowledge, no previous work in the literature has been dealt with such constrained dynamics. Only the free swing motion of the IWIP was provided and analyzed. In this work, the dynamics of the IWIP is subject to external disturbances and state constraints. Such state constraints are taken into account for the first time in the present work and play an important role in the design of the tracking controller for the self-generated limit cycle.

3. The dynamics of the IWIP is a simple class of nonlinear systems with trigonometric nonlinearities, which can occur in robotic applications, such as the single-link flexible robot manipulator [66, 67] and the pitch dynamics of a simplified helicopter model [68, 69], and other nonlinear systems.

4. Moreover, the IWIP was known as a testbed underactuated mechanical system used to design new controllers. It can be used for example to control the simplest model of a walking robot leg [70, 71]. Authors in $[70,71]$ used a liner inverted pendulum controlled via a flywheel model in the sagittal plane [70] and in the 3D [71] in order to generate angular momentum. They showed that such flywheel-based inverted pendulum can effectively describe the reaction of human if perturbed by external force while walking and standing. A related approach was employed also in [72] for the SURENA III humanoid robot using the linear inverted pendulum with a flywheel. Actually, the effect of angular momentum of the upper-body, especially the torso and arms, of a biped robot can play an important role in push 
recovery and hence walking over rough terrain. The effect can be embedded by considering the upper body as a flywheel that can be actuated directly [73].

5. Bipedal robot control techniques implementing self-clocking properties as presented in [74] have desirable properties, like the ability to drive the robot step with respect to its own geometry. In $[75,76]$, the motivation of adding a disk to the legged robot dynamics is to produce a desirable effect, a desired zero moment point, and hence to design the geometry of the walking step. A related work was presented also in [77] where authors employed the same method of reference trajectory generation introduced in [53] for the IWIP. In addition, an adaptive control of a gyroscopically stabilized pendulum and its application to a single-wheel pendulum Robot was presented in [78] using the conventional reaction wheel pendulum model.

\subsection{Objective and contributions}

In this paper, we will use the IDA-PBC introduced in $[34,35]$ to control the IWIP at its upright position. Thus, we will (briefly) show that the nonlinear dynamics of the IWIP under the IDA-PBC demonstrates a stable limit cycle through a super-critical Hopf bifurcation as some control gain of the IDA-PBC varies. Indeed, we will present stability conditions of the controlled equilibrium point with respect to the control gains. Moreover, we will develop conditions for the presence of the Hopf bifurcation and then of periodic oscillation. Furthermore, stability of these periodic oscillations is investigated numerically by means of the first Lyapunov value. Thus, we will show that for some critical control gains, the controlled dynamics of the IWIP exhibits both sub-critical and super-critical Hopf bifurcations. At this last critical bifurcation point, the IWIP under the IDA-PBC experiences a stable period-1 limit cycle. However, the existence of multiple attractors, and hence stable limit cycles, in the parameter space is a common phenomenon in nonlinear systems. Thus, depending on initial conditions, new limit cycles or other kinds of attractors (such as chaotic or quasi-periodic attractor) can be observed besides the already known, i.e. the stable limit cycle. Each limit set (attractor) is defined by its basin of attraction. For the underactuated IWIP as a physical system, it is difficult to provide the adequate initial conditions in order to guarantee that the IWIP converges to the desired stable limit cycle. Moreover, if we assume that the system trajectory converges to the desired limit cycle for all sets of initial conditions, the second problem lies in the speed (or time) of convergence itself. In this work, we show that for an initial condition almost identical to the desired one and for some value of the control gain, the trajectory of the IWIP under the IDA-PBC requires much time to converge and hence to stabilize around its own limit cycle. Moreover, we show that the two periodic motions are not synchronized. Then, the main contribution in this paper is to solve this problem for the underactuated IWIP by tracking the desired generated stable limit cycle. Our methodology lies in the tracking of a reference model as a generator of the desired limit cycle. Such reference model is the underactuated nonlinear dynamics of the IWIP under the IDA-PBC. For some set of control gains, such dynamics exhibits a stable period-1 limit cycle for a predefined initial condition. The objective is that the (physical) IWIP system tracks the desired reference model, which generates the desired stable period-1 limit cycle. To achieve this goal, our approach is to add a new control input (a limit-cycle tracking control law) to the controlled IWIP system. Thus, the limit cycle tracking problem will be reformulated as the stabilization problem of the tracking error.

According to [34, 35, 52-54], all the states of the physical device of the IWIP are available for direct measurement. Thus, in this paper, we will adopt a state-feedback control law to solve the self-generated limit cycle tracking problem of for the underactuated IWIP. Moreover, the constraints in the dynamics of the IWIP are introduced. Actually, two main keys are used in order to solve the tracking problem. The first key is the use of the S-procedure [61] in order to reduce the conservatism of the classical Lyapunov approach. The S-procedure gives in fact the possibility of reducing the field of verification conditions in a useful subspace $\Omega$, that is from $\mathbb{R}$ to some $\Omega \subset \mathbb{R}$. For the IWIP, the useful subspace is that limited with the unilateral constraints. The problem of finding stability conditions of the tracking error will be recast as a nonconvex optimization problem based on BMIs. The second key for achieving the tracking problem is the use of the Schur complement [61] and also the matrix inversion lemma in order to transform the BMIs into LMIs, which will be used to allow a numerical solution of the problem. We will show the effectiveness of the developed controller for the tracking of the desired self-generated period-1 limit cycle. We will achieve several simulations: a nominal system without uncertainties in the friction parameters and disturbance, a system under uncertain friction parameters, and a system subject to constant and randomly time-varying external disturbances. 


\subsection{Structure of the paper}

The rest of the paper is organized as follows. In Section 2, a brief description of the IWIP, its dynamics and the IDA-PBC are presented. Self-generation of a stable limit cycle through the Hopf bifurcation is briefly discussed also in this section. Problem formulation of the self-generated limit cycle tracking of the underactuated IWIP under the IDA-PBC is described in Section 3. Section 4 deals with the design of the tracking control law using the framework of LMI. Some well-known preliminaries for solving the asymptotic stabilization problem are provided first. Transformation of the BMIs into LMIs is realized in this section too. Section 5 is dedicated to simulation results. Concluding remarks are drawn in Section 6. Finally, an analysis of the dynamics of the IWIP under the IDA-PBC in order to demonstrate existence of the Hopf bifurcations and then generation of the stable limit cycle is given in Appendix.

\section{The underactuated inertia wheel inverted pendulum under the IDA-PBC}

\subsection{Description of the underactuated inertia wheel inverted pendulum}

The underactuated inertia wheel inverted pendulum (IWIP) (Fig. 1) consists of an inverted pendulum pivoting on a frictionless point with a rotating wheel on the top. Its mechanical structure is sketched in Fig. 2. In Fig. 2, the joint between the frame and the pendulum body is unactuated (passive), while the joint between the body and the inertia wheel is actuated (active) via the control input $u$. The inertia wheel is free to rotate about an axis parallel to the axis of rotation of the pendulum. Hence, the IWIP is a planar mechanical system. This electromechanical system is controlled by a DC motor actuating the inertia wheel (see [31] for further descriptions of this system). The motor torque produces an angular acceleration of the rotating wheel, which generates a torque acting on the passive joint of the pendulum by means of the dynamic coupling. Therefore, this passive joint can be controlled through the acceleration of the inertia wheel. The dynamic parameters of the IWIP and its description are given in Table 1. In Fig. $2, \theta_{1}$ is the angular position of the pendulum body, whereas $\theta_{2}$ is the rotation angle of the inertia wheel. The motion of the pendulum is subject to unilateral constraints defined as:

$$
\Omega=\left\{\theta_{1} \in \mathbb{R}:-\sigma \leq \theta_{1} \leq \sigma\right\} .
$$

where $\sigma=\theta_{1 \max }$. According to [53], the pendulum angle value is approximately $\pm 10^{\circ}$ at standstill (i.e. at the pendulum stop). Then, $\sigma=10^{\circ}$.

In this work, we ignore the collision effect of the pendulum body with the stop.

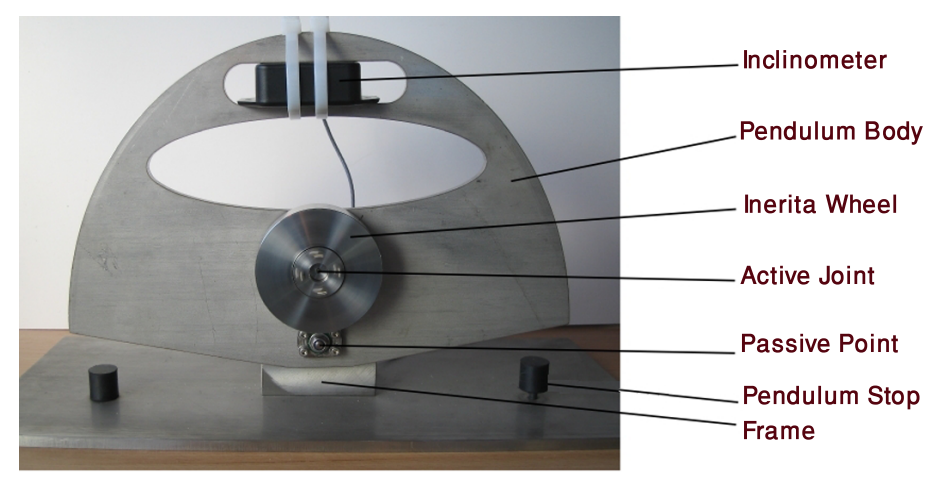

Fig. 1 Physical device of the underactuated inertia wheel inverted pendulum.

\subsection{Dynamic model of the IWIP}

The dynamic model of the underactuated IWIP is obtained by application of the Euler-Lagrange formulation [79]. Based on [34, 35, 53], the mathematical model of the IWIP augmented with viscous frictions in the active and passive joints and with disturbances is described by: 


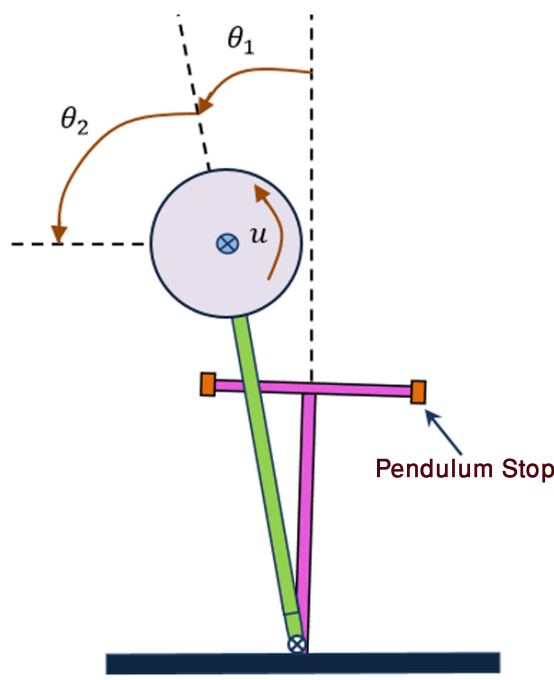

Fig. 2 Schematic representation of the underactuated inertia wheel inverted pendulum.

Table 1 Important dynamic parameters of the underactuated inertia wheel inverted pendulum.

\begin{tabular}{llll}
\hline Symbol & Description & Value & Unit \\
\hline$m_{1}$ & Pendulum mass & 3.228 & $\mathrm{Kg}$ \\
$m_{2}$ & Wheel mass & 0.33081 & $\mathrm{Kg}$ \\
$l_{1}$ & Pendulum center of mass position & 0.06 & $\mathrm{~m}$ \\
$l_{2}$ & Wheel center of mass position & 0.044 & $\mathrm{~m}$ \\
$I_{1}$ & Pendulum inertia & 0.0314 & $\mathrm{Kg} \cdot \mathrm{m}^{2}$ \\
$I_{2}$ & Wheel inertia & $4.176 e-4$ & $\mathrm{Kg} \cdot \mathrm{m}^{2}$ \\
\hline
\end{tabular}

$$
\left[\begin{array}{cc}
I+I_{2} & I_{2} \\
I_{2} & I_{2}
\end{array}\right]\left[\begin{array}{c}
\ddot{\theta}_{1} \\
\ddot{\theta}_{2}
\end{array}\right]-\left[\begin{array}{c}
b g \sin \left(\theta_{1}\right) \\
0
\end{array}\right]+\left[\begin{array}{c}
\delta_{1} \dot{\theta}_{1} \\
\delta_{2} \dot{\theta}_{2}
\end{array}\right]=\left[\begin{array}{l}
0 \\
1
\end{array}\right] u+\left[\begin{array}{l}
1 \\
0
\end{array}\right] \zeta
$$

where $\dot{\theta}_{1}$ and $\dot{\theta}_{2}$ correspond to the velocity of the pendulum body and the inertia wheel, respectively; $\ddot{\theta}_{1}$ and $\ddot{\theta}_{2}$ are their corresponding accelerations; $u$ is the torque applied by the motor on the inertia wheel; $\zeta$ is the external disturbing torque applied to the pendulum; $I=m_{1} l_{1}^{2}+m_{2} l_{2}^{2}+I_{1} ; b=m_{1} l_{1}+m_{2} l_{2} ; \delta_{1}$ and $\delta_{2}$ represent, respectively, the friction coefficients of the passive and the active articulations.

According to [31, 34, 35, 53], the physical device of the IWIP system (Fig. 1) was mechanically designed so as to minimize the effects of viscous frictions. Thus, in this study the frictions of the active and passive joints, i.e. $\delta_{1}$ and $\delta_{2}$, are so low that we have neglected. Then, in the sequel of this work, we consider the nominal system, that is without friction $\left(\delta_{1}=\delta_{2}=0\right)$. Moreover, the torque $\zeta$ is considered to be zero, that is the nominal system is not subject to external disturbing torque. Hence, the nominal dynamics of the IWIP is described as follows:

$$
\left[\begin{array}{cc}
I+I_{2} & I_{2} \\
I_{2} & I_{2}
\end{array}\right]\left[\begin{array}{l}
\ddot{\theta}_{1} \\
\ddot{\theta}_{2}
\end{array}\right]-\left[\begin{array}{c}
b g \sin \left(\theta_{1}\right) \\
0
\end{array}\right]=\left[\begin{array}{l}
0 \\
1
\end{array}\right] u
$$

Remark 1 The effect of the friction coefficients $\delta_{1}$ and $\delta_{2}$ and the external disturbance $\zeta$ on the tracking problem of the self-generated limit cycle of the underactuated IWIP under IDA-PBC will be analyzed in section 5 through numerical simulations. The damping coefficients $\delta_{1}$ and $\delta_{2}$ will be considered as two uncertain parameters.

Authors in $[25,34,35]$ introduced the following change of coordinates:

$$
\left[\begin{array}{l}
q_{1} \\
q_{2}
\end{array}\right]=\left[\begin{array}{ll}
1 & 0 \\
1 & 1
\end{array}\right]\left[\begin{array}{l}
\theta_{1} \\
\theta_{2}
\end{array}\right]
$$

Thus, using (4), the dynamics in (3) can be rewritten like so: 


$$
\left[\begin{array}{cc}
I & 0 \\
0 & I_{2}
\end{array}\right]\left[\begin{array}{l}
\ddot{q}_{1} \\
\ddot{q}_{2}
\end{array}\right]-\left[\begin{array}{c}
b g \sin \left(q_{1}\right) \\
0
\end{array}\right]=\left[\begin{array}{c}
-1 \\
1
\end{array}\right] u
$$

The model (5) can be rewritten through Hamilton's equations of motion as [24, 34, 35]:

$$
\left[\begin{array}{c}
\dot{q}_{1} \\
\dot{q}_{2} \\
\dot{p}_{1} \\
\dot{p}_{2}
\end{array}\right]=\left[\begin{array}{c}
\frac{p_{1}}{I} \\
\frac{p_{2}}{I_{2}} \\
b g \sin \left(q_{1}\right)-u \\
u
\end{array}\right]
$$

where $p_{1}=I \dot{q}_{1}$ and $p_{2}=I_{2} \dot{q}_{2}[25,34,35]$.

\subsection{The IDA-PBC controller}

According to $[25,27,34,35]$, the Interconnection and Damping Assignment-Passivity Based Control (IDA$\mathrm{PBC}$ ) is expressed as follows:

$$
u=\gamma \sin \left(q_{1}\right)+k_{1} q_{1}+k_{2} q_{2}+k_{3} p_{1}+k_{4} p_{2}
$$

with $k_{i}$ are control gains and $k_{i}>0, \forall i=1,2,3,4$, and $\gamma>b g$.

In the expression of the IDA-PBC $u(7)$, the first three terms reveal the energy shaping control to assign the equilibrium. However, the last two terms inject damping to the pendulum body and the inertia wheel to achieve asymptotic stability. In [34, 35], the control gains were calculated and selected as: $\gamma=6.1284$, $k_{1}=1.0367, k_{2}=0.0011, k_{3}=16.62$ and $k_{4}=3.4640$. With these parameters and under the dynamic nonlinear output feedback IDA-PBC (7), the underactuated IWIP was stabilized in its upward equilibrium point.

\subsection{Self-generation of a stable limit cycle through Hopf bifurcation}

By introducing (7) in (6), the closed-loop dynamics of the underactuated IWIP is given by:

$$
\left[\begin{array}{c}
\dot{x}_{1} \\
\dot{x}_{2} \\
\dot{x}_{3} \\
\dot{x}_{4}
\end{array}\right]=\left[\begin{array}{c}
\frac{x_{3}}{I} \\
\frac{x_{4}}{I_{2}} \\
(b g-\gamma) \sin \left(x_{1}\right)-k_{1} x_{1}-k_{2} x_{2}-k_{3} x_{3}-k_{4} x_{4} \\
\gamma \sin \left(x_{1}\right)+k_{1} x_{1}+k_{2} x_{2}+k_{3} x_{3}+k_{4} x_{4}
\end{array}\right]
$$

with $\boldsymbol{x}=\left[\begin{array}{llll}x_{1} & x_{2} & x_{3} & x_{4}\end{array}\right]^{\mathrm{T}}=\left[\begin{array}{llll}q_{1} & q_{2} & p_{1} & p_{2}\end{array}\right]^{\mathrm{T}}$.

It is straightforward to demonstrate that the equilibrium point of the nonlinear system (8) is expressed by:

$$
\boldsymbol{x}_{e q}=\left[\begin{array}{c}
k \pi \\
-k \pi \frac{k_{1}}{k_{2}} \\
0 \\
0
\end{array}\right]
$$

where $k \in \mathbb{Z}$.

Moreover, as the controlled IWIP is under the state constraints (1), then the only equilibrium point is the origin. The Jacobian matrix around the equilibrium point $\boldsymbol{x}_{e q}$ (we consider only the upright position) of the closed-loop nonlinear system (8) is defined as follows:

$$
\boldsymbol{J}=\left[\begin{array}{cccc}
0 & 0 & \frac{1}{I} & 0 \\
0 & 0 & 0 & \frac{1}{I_{2}} \\
b g-\gamma-k_{1} & -k_{2} & -k_{3} & -k_{4} \\
\gamma+k_{1} & k_{2} & k_{3} & k_{4}
\end{array}\right]
$$

It is worth mentioning that as the only equilibrium point of the system (8) is the origin, then the only way for which such equilibrium point will lose its stability is through the Hopf bifurcation. Indeed, by varying the control gains $k_{i}$ and by analyzing the eigenvalues of the Jacobian matrix (10), the controlled equilibrium point $\boldsymbol{x}_{e q}$ loses its stability at some critical values of the gains $k_{i}$. At these critical values, saying $k_{i}^{c}$, the closed-loop nonlinear dynamics (8) undergoes a Hopf bifurcation, at which there is a creation of 
either a stable limit cycle or an unstable one. Since the unstable limit cycle (as an unstable solution in nonlinear dynamics) is not observable, and hence only the stale limit cycle has physical means.

We recall that the main objective of this paper is the design of a tracking controller of some selfgenerated stable limit cycle of the IWIP for some set of gain parameters $k_{i}$. Then, stability investigation of the equilibrium point $\boldsymbol{x}_{e q}$ with respect to the parameters $k_{i}$, existence demonstration of the Hopf bifurcation and then limit cycles and its stability are not developed in this part and only presented in Appendix 1. A more detailed work dealing with this subject will be developed in another paper.

According to Appendix 1, by fixing the control gains $k_{1}, k_{2}$ and $k_{3}$, and by varying the remaining one $k_{4}$, the Hopf bifurcation occurs at two critical values of $k_{4}: k_{4,1}^{c} \approx 0.8716$ and $k_{4,2}^{c} \approx 27.1977$. For $k_{4,1}^{c}$, the Hopf bifurcation was found to be sub-critical and hence only unstable limit cycles were born. However, for the second critical value $k_{4,2}^{c}$, the Hopf bifurcation is super-critical and accordingly a period-1 stable limit cycle was born. For $k_{4}$ lying between $k_{4,1}^{c}$ and $k_{4,2}^{c}$, the equilibrium point is asymptotically stable.

In order to investigate the dynamic behavior of the IWIP as the parameter $k_{4}$ varies, we used bifurcation diagrams where some measure (for example $x_{1}$, i.e. $\theta_{1}$ ) of the solution state is plotted against the parameter $k_{4}$. Moreover, as the origin is the only equilibrium point, then we will focus on the generated stable limit cycle through the super-critical Hopf bifurcation. Figure 3 shows a bifurcation diagram of the controlled underactuated IWIP as the parameter $k_{4}$ varies. For $k_{4}<k_{4,2}^{c}$, the controlled IWIP converges to the stable equilibrium point (marked as SEP in Fig. 3). As $k_{4}$ increases, the SEP loses its stability and hence becomes an unstable focus (indicated as UEP) via the Hopf bifurcation (marked as HB in Fig. 3). At the critical point $k_{4,2}^{c} \approx 27.1977$, the controlled IWIP experiences a stable period-1 limit cycle. As $k_{4}$ increases, the amplitude of this limit cycle grows rapidly (according to a certain square function). Thus, the stable limit cycle persists for a small interval of the control gain $k_{4}$, i.e. $k_{4,2}^{c}<k_{4}<30$.

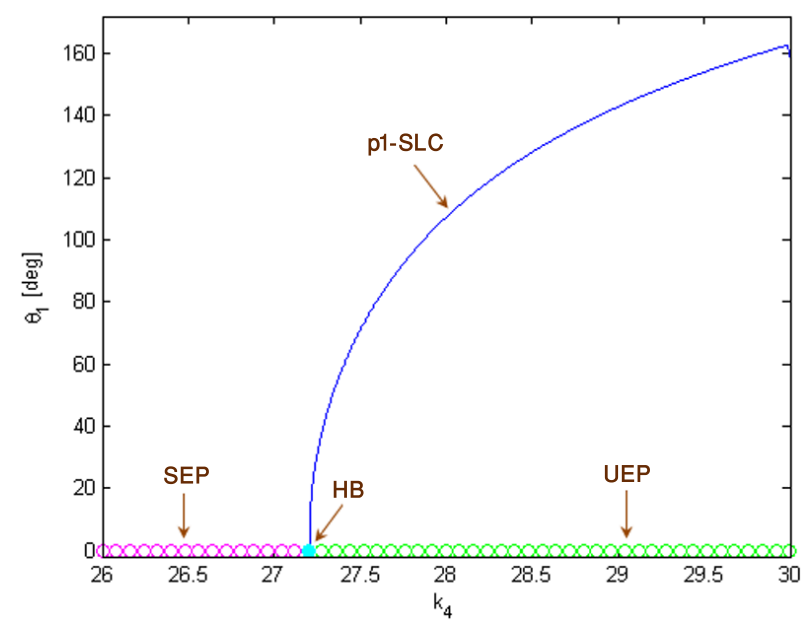

Fig. 3 Bifurcation diagram displaying the steady solutions of the closed-loop unconstrained IWIP as the control gain $k_{4}$ varies. This diagram shows the maximum angular position, $\theta_{1}=q_{1}=x_{1}$, of the pendulum body versus $k_{4}$.

Figure 4(a) (resp. Figure 4(b)) reveals some stable period-1 limit cycles in the phase portrait of the pendulum (resp. the inertia wheel) for some values of the parameter $k_{4}$. The smallest limit cycle is depicted for $k_{4}=27.201$ and the largest one is plotted for $k_{4}=27.25$. For $k_{4}=27.201$, the pendulum body reaches the angular position about $9^{\circ}$. Whereas, for $k_{4}=27.25$, the maximum angular position of the pendulum is about $30^{\circ}$. It is obvious how the limit cycle of the pendulum grows in terms of amplitude as the control gain $k_{4}$ increases slightly.

We emphasize that stable limit cycles in the bifurcation diagram in Fig. 3 are computed using an iterative method called as the Poincaré shooting method [80] by defining first a Poincaré section:

$$
\mathcal{P}_{\mathcal{S}}=\left\{\boldsymbol{x} \in \mathbb{R}^{4 \times 1}, h(\boldsymbol{x})=x_{3}=\mathcal{C}_{\mathcal{S}} \boldsymbol{x}=0, \quad \mathcal{C}_{\mathcal{S}} \dot{\boldsymbol{x}}<0\right\}
$$

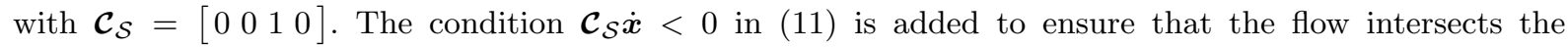
hyperplane defined by $\mathcal{C}_{\mathcal{S}} \boldsymbol{x}=0$ in only one direction.

It is worth noting that for a stable period-1 limit cycle, the flow starting from its fixed point $x_{\star}$ located on the Poincaré section (11) returns to the Poincaré section and intersects it in the same fixed point $x_{\star}$. The return time, say $\tau_{r}$, represents the time between two successive intersections with the Poincaré section 


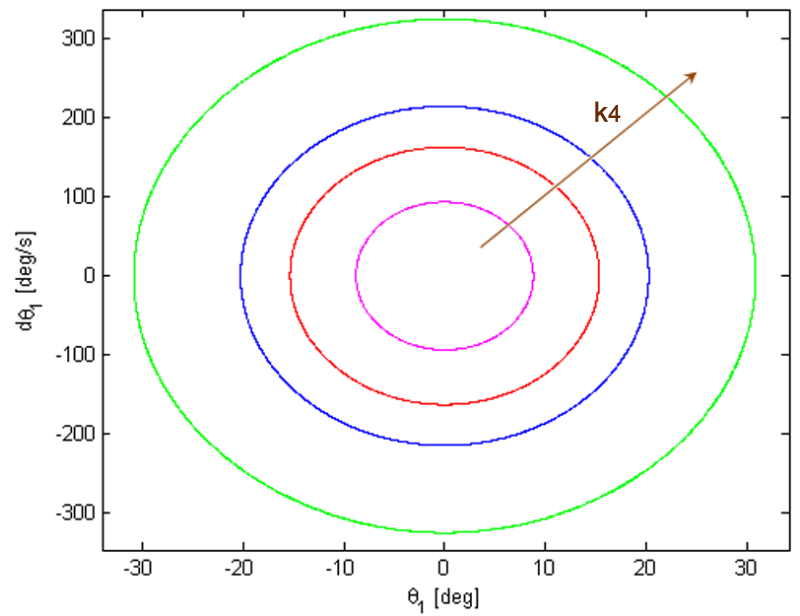

(a)

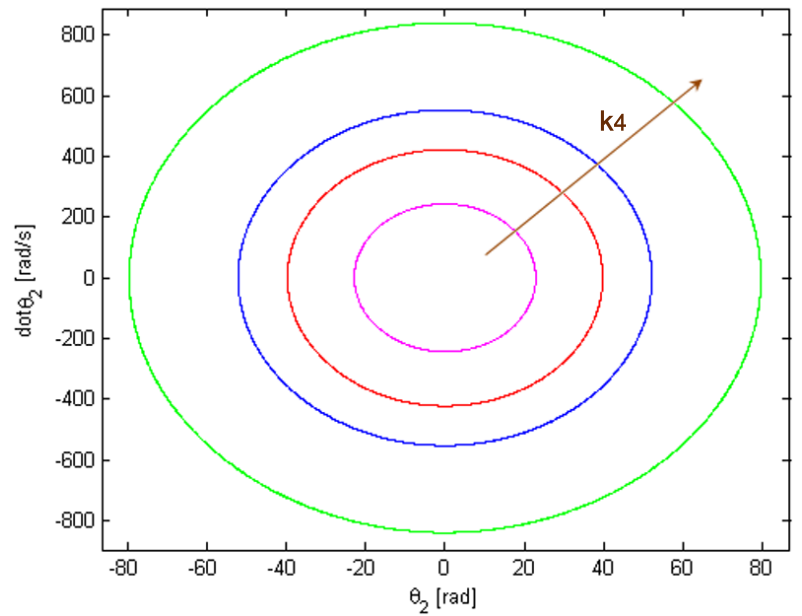

(b)

Fig. 4 Limit cycles of the pendulum for some values of the control gain parameter $k_{4}$. From inside to outside: $k_{4}=27.201$, $k_{4}=27.21, k_{4}=27.22$ and $k_{4}=27.25$. (a) shows the limit cycles of the pendulum body, whereas (b) displays the limit cycles of the inertia wheel.

and it defines the period of the stable period-1 limit cycle. We note that the return time is calculated when integrating the nonlinear dynamics (8) and hence locating the fixed point $x_{\star}$ through the Shooting method. For example, the smallest stable limit cycle, i.e. for $k_{4}=27.201$, has a self-sustained oscillation period about $0.5900[s]$. However, for $k_{4}=27.25$, the self-sustained oscillation period is about $0.5989[s]$. We stress that the first value of the period (i.e. for $k_{4}=27.201$ ) is almost identical to that calculated theoretically through expression (91) (see Appendix 1).

\section{Problem formulation}

We emphasize that the bifurcation diagram in Fig. 3 and the limit cycles in Fig. 4 are plotted without taking into account the constraints on the motion of the pendulum body of the IWIP. We note that in some previous papers such as that of Ortega and his co-workers, the motion of the IWIP was totally free and no constraint was considered. Then, if we consider here that the motion of the IWIP is subject to unilateral constraints defined by (1) and as presented in Fig. 1 and Fig. 2, some limit cycles in Fig. 3 and in Fig. 4 will not be considered. The maximum angular position that can be reached by the pendulum body is about $10^{\circ}$. Then, in the sequel of this paper, we will interest only in the first stable limit cycle in Fig. 4(a), i.e. for $k_{4}=27.201$. This stable limit cycle will be used as a reference trajectory, which was generated via the nonlinear dynamics (8) under the IDA-PBC. The initial condition of such limit cycle is chosen to be: $\left(\theta_{1}^{r}(0), \theta_{2}^{r}(0), \dot{\theta}_{1}^{r}(0), \dot{\theta}_{2}^{r}(0)\right)=\left(-8.8579^{\circ}, 23.0117 \mathrm{rad}, 0.0066 \mathrm{rad} / \mathrm{s},-0.9831 \mathrm{rad} / \mathrm{s}\right)$.

We want to show the motion of the IWIP under the IDA-PBC for the same gain parameter $k_{4}=27.201$ and for another initial condition: $\left(\theta_{1}(0), \theta_{2}(0), \dot{\theta}_{1}(0), \dot{\theta}_{2}(0)\right)=\left(-10^{\circ}, 0^{\circ}, 0 \mathrm{rad} / \mathrm{s}, 0 \mathrm{rad} / \mathrm{s}\right)$. The choice of $\theta_{1}(0)$ is motivated by the physical properties of the IWIP since simulation is started from the rest position at standstill, for which $\theta_{1}(0)= \pm 10^{\circ}$. Here, we chosen $\theta_{1}(0)=-10^{\circ}$ in order that such initial position of the pendulum body is almost identical to that of the desired one $\theta_{1}^{r}(0)$. Figure 5 shows the temporal evolution of both the desired (reference) angular position $\theta_{1}^{r}$ and the angular position $\theta_{1}$ of the pendulum body. The numerical simulation was achieved for $500[s]$. Figure 5 (a) reveals behavior of the IWIP during the first three seconds, whereas Fig. 5(b) depicts the motion during the last three seconds. It is obvious that for almost two identical initial positions of the pendulum body, the motion of the IWIP diverges progressively in time and the IWIP becomes in phase lag with respect to the reference. Furthermore, even after the stabilization of the motion of the IWIP, the resulting behavior is found to be completely different to the desired one, where the two motions are not synchronized.

It is worth noting that such result is very meaningful from chaos and bifurcation theory. Indeed, for $k_{4}>k_{4,2}^{c}$ the limit set is the stable period-1 limit cycle. By decreasing the value of the bifurcation parameter $k_{4}$, one among the eigenvalues of the Jacobian matrix of the Poincaré map associated to the stable limit cycle becomes very near to the boundary of the unit circle. Thus, robustness of the stable period- 1 stable limit cycle towards perturbations decreases. Hence the limit cycle becomes very sensitive to weak perturbation 


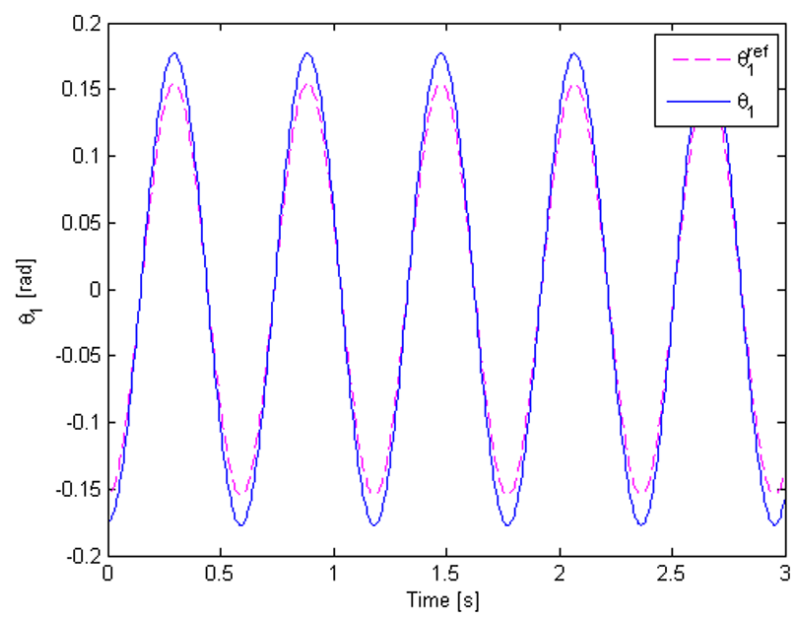

(a)

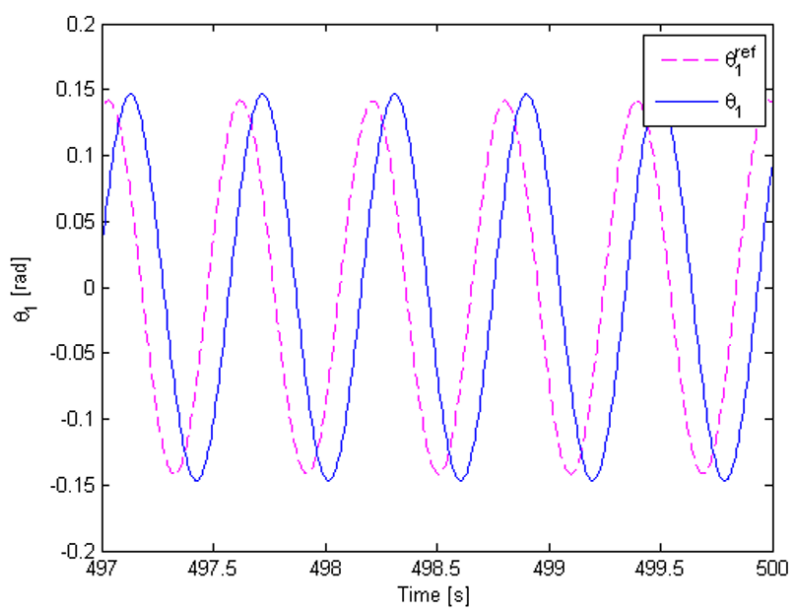

(b)

Fig. 5 Behavior of the IWIP under IDA-PBC for the gain parameter $k_{4}=27.201$ and for neighborhood initial conditions. Here, only the motion of the angular position is depicted. The trajectory $\theta_{1}^{r e f}$ is the desired one and which reveals the stable limit cycle. However, $\theta_{1}$ represents the trajectory of the IWIP for another initial condition. (a) shows the motion for the first three seconds, and (b) shows the motion during the last three seconds.

around it. As a result, for a neighborhood initial condition, the system trajectory needs a long time in order to converge to the stable period-1 limit cycle.

Our objective is then to solve such problem by synchronizing the periodic motion of the IWIP with the desired self-sustained oscillation, that is with the desired stable limit cycle. Thus, this self-generated stable limit cycle will be used as a reference trajectory, which was generated via the nonlinear dynamics (8) under the IDA-PBC. In other words, the controlled nonlinear dynamics (8) will be considered as a reference model to track.

Relying on (8), the nonlinear dynamics of the IWIP under the unilateral constraints can be reformulated as:

$$
\dot{\boldsymbol{x}}=\boldsymbol{J} \boldsymbol{x}+\boldsymbol{D} f\left(x_{1}\right) \text { where }-\sigma \leq x_{1} \leq \sigma,
$$

with $\boldsymbol{D}=\left[\begin{array}{c}0 \\ 0 \\ b g-\gamma \\ \gamma\end{array}\right]$, and $f(x)=\sin (x)-x$.

It is straightforward to demonstrate that:

$$
\sin (\sigma)-\sigma \leq f(x) \leq \sigma-\sin (\sigma) \text { for all }-\sigma \leq x \leq \sigma,
$$

The objective is then to add a control input $v$ to the nonlinear dynamics (8) of the IWIP to track the self-generated period-1 stable limit cycle. Such additional control input $v$ is added first to the original nonlinear dynamics (2). Thus, we obtain the following new dynamic system of the IWIP:

$$
\left[\begin{array}{cc}
I+I_{2} & I_{2} \\
I_{2} & I_{2}
\end{array}\right]\left[\begin{array}{l}
\ddot{\theta}_{1} \\
\ddot{\theta}_{2}
\end{array}\right]-\left[\begin{array}{c}
b g \sin \left(\theta_{1}\right) \\
0
\end{array}\right]+\left[\begin{array}{l}
\delta_{1} \dot{\theta}_{1} \\
\delta_{2} \dot{\theta}_{2}
\end{array}\right]=\left[\begin{array}{l}
0 \\
1
\end{array}\right] u+\left[\begin{array}{l}
0 \\
1
\end{array}\right] v+\left[\begin{array}{l}
1 \\
0
\end{array}\right] \zeta
$$

According to previous results, system (12) and nonlinear dynamics (14), the dynamics of the controlled IWIP under the additional control input $v$ can be recast as:

$$
\dot{\boldsymbol{x}}=\boldsymbol{J} \boldsymbol{x}+\boldsymbol{D} f\left(x_{1}\right)+\boldsymbol{B} v \text { where }-\sigma \leq x_{1} \leq \sigma,
$$

with $\boldsymbol{B}=\left[\begin{array}{c}0 \\ 0 \\ -1 \\ 1\end{array}\right]$

The objective is then to design the controller $v$ in order to track the one-periodic stable limit cycle, which is generated via the following reference model: 


$$
\dot{\boldsymbol{x}}^{r}=\boldsymbol{J} \boldsymbol{x}^{r}+\boldsymbol{D} f\left(x_{1}^{r}\right) \text { where }-\sigma \leq x_{1}^{r} \leq \sigma,
$$

Remark 2 Relying on the dynamics (2) of the underactuated IWIP with parametric uncertainties in the two friction coefficients $\delta_{1}$ and $\delta_{2}$ and subject to the external disturbance $\zeta$, the IDA-PBC $u$ in (7) and according to the system (15), the state space representation of the controlled IWIP under the parametric uncertainties and the external disturbance can be reformulated as follows:

$$
\dot{\boldsymbol{x}}=\boldsymbol{J} \boldsymbol{x}+\boldsymbol{D} f\left(x_{1}\right)+\boldsymbol{B} v+\tilde{\boldsymbol{J}} \boldsymbol{x}+\tilde{\boldsymbol{D}} \zeta \text { where }-\sigma \leq x_{1} \leq \sigma,
$$

where $\tilde{\boldsymbol{J}}=\left[\begin{array}{cccc}0 & 0 & 0 & 0 \\ 0 & 0 & 0 & 0 \\ 0 & 0 & -\frac{\delta_{1}+\delta_{2}}{I} & \frac{\delta_{2}}{I_{2}} \\ 0 & 0 & \frac{\delta_{2}}{I} & -\frac{\delta_{2}}{I_{2}}\end{array}\right]$ and $\tilde{\boldsymbol{D}}=\left[\begin{array}{l}0 \\ 0 \\ 1 \\ 0\end{array}\right]$

Thus, our goal is to design the new control law $v$ such that the constrained nonlinear system (15) of the controlled IWIP tracks the reference model (16). The tracking error, $e=\boldsymbol{x}-\boldsymbol{x}^{r}$, between the nonlinear dynamics (15) and the reference model (16) is defined as:

$$
\dot{\boldsymbol{e}}=\boldsymbol{J} \boldsymbol{e}+\boldsymbol{D}\left(f\left(x_{1}\right)-f\left(x_{1}^{r}\right)\right)+\boldsymbol{B} v \text { where }-2 \sigma \leq e_{1} \leq 2 \sigma,
$$

with $e_{1}=x_{1}-x_{1}^{r}$.

Therefore, our approach for achieving the tracking problem lies in the design of the control law $v$ stabilizing the tracking error of the nonlinear system (18), which is the objective of the next section.

Remark 3 The nonlinear dynamics (12) of the IWIP is under state constraints in which the motion of pendulum or the state $x_{1}=\theta_{1}$ evolves in a small region (1) defined as $\left|x_{1}\right| \leq 10^{\circ}$. Moreover, the nonlinear term $f\left(x_{1}\right)$ presented in the dynamics of the IWIP depends only on the state $x_{1}, f\left(x_{1}\right)=\sin \left(x_{1}\right)-x_{1}$. Thus, it is possible to use the approximated linear model instead of the nonlinear model to solve the tracking control problem. In this case, we will have $f\left(x_{1}\right)=0$ since $\sin \left(x_{1}\right) \approx x_{1}$. Hence, the error dynamics in (18) becomes

$$
\dot{\boldsymbol{e}}=\boldsymbol{J} \boldsymbol{e}+\boldsymbol{B} v \text { where }-2 \sigma \leq e_{1} \leq 2 \sigma
$$

It is worth to note that there is no remarkable difference between the obtained results using the nonlinear system (18) or the linear system (19). However, the strategy developed in this work for the controller synthesis by considering the nonlinear dynamics (18) can be applied for more general constrained nonlinear systems for which the states evolve in a large region as for the case of the single-link rotary flexible joint robot studied in [67].

\section{Design of the tracking control law}

The tracking control law is adopted to be defined like so:

$$
v=-k_{2} e_{2}+w
$$

with $k_{2}$ is the control gain in the IDA-PBC (7), $e_{2}=x_{2}-x_{2}^{r}$, and $w$ is an auxiliary tracking control law.

By substituting expression of $v$ in the dynamics (18), it follows that the second state variable $e_{2}$ does not affect any other variable. Therefore, there is no interest on its evolution and hence it can be discarded. Then, the nonlinear dynamics (18) can be simplified as:

$$
\dot{\overline{\boldsymbol{e}}}=\boldsymbol{A} \overline{\boldsymbol{e}}+\overline{\boldsymbol{D}}\left(f\left(x_{1}\right)-f\left(x_{1}^{r}\right)\right)+\overline{\boldsymbol{B}} w \text { where }-2 \sigma \leq e_{1} \leq 2 \sigma,
$$

with $\overline{\boldsymbol{e}}=\left[\begin{array}{lll}e_{1} & e_{3} & e_{4}\end{array}\right]^{\mathrm{T}}, \boldsymbol{A}=\left[\begin{array}{ccc}0 & \frac{1}{I} & 0 \\ b g-\gamma-k_{1} & -k_{3} & -k_{4} \\ \gamma+k_{1} & k_{3} & k_{4}\end{array}\right], \overline{\boldsymbol{D}}=\left[\begin{array}{c}0 \\ b g-\gamma \\ \gamma\end{array}\right]$, and $\overline{\boldsymbol{B}}=\left[\begin{array}{c}0 \\ -1 \\ 1\end{array}\right]$.

Thus, the tracking problem will be recast as an asymptotic stability problem of the tracking error $\bar{e}$ of the nonlinear system (21) by means of the control law $w$. To achieve such asymptotic stability, we adopt a simple state-feedback linear control law as:

$$
w=\left\{\begin{array}{l}
\boldsymbol{K} \overline{\boldsymbol{e}} \text { whenever }\|\overline{\boldsymbol{e}}\|>\epsilon \\
0 \quad \text { elsewhere }
\end{array}\right.
$$


where $\boldsymbol{K}$ is a constant gain matrix which will be obtained later, and $\epsilon$ is very small positive constant.

Substituting this control law into (21), it yields the new closed-loop dynamics:

$$
\dot{\overline{\boldsymbol{e}}}=(\boldsymbol{A}+\overline{\boldsymbol{B}} \boldsymbol{K}) \overline{\boldsymbol{e}}+\overline{\boldsymbol{D}}\left(f\left(x_{1}\right)-f\left(x_{1}^{r}\right)\right) \text { where }-2 \sigma \leq e_{1} \leq 2 \sigma \text { and }\|\overline{\boldsymbol{e}}\|>\epsilon,
$$

The problem lies then in the stabilization of the nonlinear dynamics (23) by designing the gain matrix $\boldsymbol{K}$. Designing the gain matrix $\boldsymbol{K}$ of the control law (22) stabilizing the nonlinear system (23) can be realized though the classical Lyapunov method. However, this method requires that the error state $\overline{\boldsymbol{e}}$ belong to the complete state space $\mathbb{R}^{3}$ (here, the nonlinear system (23) is three-dimensional). However, for our stabilization problem, the error state $\overline{\boldsymbol{e}}$ is constrained and hence it belongs to a certain subspace $\Omega$ in $\mathbb{R}^{3}$. Then, using the Lyapunov method, the stability conditions must be only verified in the working state space $\Omega$ and not in $\mathbb{R}$. As a result, this will give stability conditions less conservative. Furthermore, looking for stability conditions in the subspace $\Omega \subset \mathbb{R}^{3}$ can conduct to obtain a control effort that is less than that in $\mathbb{R}^{3}$. Therefore, our methodology to solve the tracking problem hinges mainly on the use of the S-procedure [61] in order to reduce the conservatism of the classical Lyapunov approach.

The S-procedure Lemma and other preliminaries are defined next.

\subsection{Preliminary Lemmas}

Lemma 1 the Young relation [81-83]

Given constant matrices $\boldsymbol{X}$ and $\boldsymbol{Y}$ with appropriate dimensions and any positive symmetric matrix $\boldsymbol{M}$, the following inequality holds:

$$
\boldsymbol{X}^{\mathrm{T}} \boldsymbol{Y}+\boldsymbol{Y}^{\mathrm{T}} \boldsymbol{X} \leq \boldsymbol{X}^{\mathrm{T}} \boldsymbol{M} \boldsymbol{X}+\boldsymbol{Y}^{\mathrm{T}} \boldsymbol{M}^{-1} \boldsymbol{Y}
$$

Lemma 2 The matrix inversion Lemma [84, 85]

Given invertible matrices $\boldsymbol{X}$ and $\boldsymbol{Y}$ such that $\boldsymbol{X} \in \mathbb{R}^{n \times n}$ and $\boldsymbol{Y} \in \mathbb{R}^{m \times m}$. Moreover, given matrices $\boldsymbol{U}$ and $\boldsymbol{V}$ with appropriate dimensions: $\boldsymbol{U} \in \mathbb{R}^{n \times m}$ and $\boldsymbol{V} \in \mathbb{R}^{m \times n}$. Then, the matrix inversion Lemma (known also as the Woodbury matrix identity) is:

$$
(\boldsymbol{X}+\boldsymbol{U} \boldsymbol{Y} \boldsymbol{V})^{-1}=\boldsymbol{X}^{-1}-\boldsymbol{X}^{-1} \boldsymbol{U}\left(\boldsymbol{Y}^{-1}+\boldsymbol{V} \boldsymbol{X}^{-1} \boldsymbol{U}\right)^{-1} \boldsymbol{V} \boldsymbol{X}^{-1}
$$

Lemma 3 The Schur complement Lemma [61]

Given matrices $\boldsymbol{Q}=\boldsymbol{Q}^{\mathrm{T}}, \boldsymbol{R}=\boldsymbol{R}^{\mathrm{T}}$ and $\boldsymbol{S}$ with appropriate dimensions, the following propositions are equivalent:

$$
\begin{gathered}
{\left[\begin{array}{cc}
\boldsymbol{Q} & \boldsymbol{S} \\
\boldsymbol{S}^{\mathrm{T}} & \boldsymbol{R}
\end{array}\right]>0} \\
\left\{\begin{array}{l}
\boldsymbol{R}>0 \\
\boldsymbol{Q}-\boldsymbol{S}^{-1} \boldsymbol{S}^{\mathrm{T}}>0
\end{array}\right. \\
\left\{\begin{array}{l}
\boldsymbol{Q}>0 \\
\boldsymbol{R}-\boldsymbol{S}^{\mathrm{T}} \boldsymbol{Q}^{-1} \boldsymbol{S}>0
\end{array}\right.
\end{gathered}
$$

Lemma 4 The S-procedure Lemma $[61,86]$

Let $\boldsymbol{F}_{0}, \ldots, \boldsymbol{F}_{p} \in \mathbb{R}^{n \times n}$ be symmetric matrices. We consider the following condition on $\boldsymbol{F}_{0}, \ldots, \boldsymbol{F}_{p}$ :

$$
\boldsymbol{\zeta}^{\mathrm{T}} \boldsymbol{F}_{0} \boldsymbol{\zeta}>0 \text { for all } \boldsymbol{\zeta} \neq \mathbf{0} \text { such that } \boldsymbol{\zeta}^{\mathrm{T}} \boldsymbol{F}_{i} \boldsymbol{\zeta} \geq 0, \quad i=1, \ldots, p
$$

If

$$
\text { there exist scalar variables } \tau_{1} \geq 0, \ldots, \tau_{p} \geq 0 \text { such that } \boldsymbol{F}_{0}-\sum_{i=1}^{p} \tau_{i} \boldsymbol{F}_{i}>0 \text {, }
$$

then (27) holds.

Remark 4 In the sequel of the paper, $(\star)$ denotes a transposed symmetric element. For example, $\left[\begin{array}{cc}\boldsymbol{X} & \boldsymbol{Y} \\ (\star) & \boldsymbol{Z}\end{array}\right]=$ $\left[\begin{array}{cc}\boldsymbol{X} & \boldsymbol{Y} \\ \boldsymbol{Y}^{\mathrm{T}} & \boldsymbol{Z}\end{array}\right]$, and $\boldsymbol{X}+(\star)=\boldsymbol{X}+\boldsymbol{X}^{\mathrm{T}}$. Moreover, $\mathcal{O}$ and $\mathcal{I}$ denote the zero matrix and the identity matrix, respectively, with appropriate dimension. 


\subsection{Asymptotic stabilization of the tracking error}

In this section, we derive conditions on asymptotic stability of the constrained nonlinear dynamics (23) of the tracking error. We state then the following theorem.

Theorem 1 If there exist a matrix $\boldsymbol{P}=\boldsymbol{P}^{\mathrm{T}}$, a matrix $\boldsymbol{K}$, some positive constants $\mu_{1}, \mu_{2}, \mu_{3}, \eta_{1}, \eta_{2}$ and $\eta_{3}$, and a matrix $\boldsymbol{M}=\boldsymbol{M}^{\mathrm{T}}>0$ such that the following matrix inequalities

$$
\begin{gathered}
{\left[\begin{array}{cc}
\boldsymbol{P}-\mu_{3} \boldsymbol{I} & \left(\mu_{1}-\mu_{2}\right) \boldsymbol{C} \\
(\star) & \epsilon \mu_{3}-4 \sigma\left(\mu_{1}+\mu_{2}\right)
\end{array}\right]>0} \\
{\left[\begin{array}{cc}
\boldsymbol{P} \boldsymbol{A}+\boldsymbol{P} \overline{\boldsymbol{B}} \boldsymbol{K}+(\star)+\boldsymbol{P} \boldsymbol{M} \boldsymbol{P}+\eta_{3} \boldsymbol{\mathcal { I }} & -\left(\eta_{1}-\eta_{2}\right) \boldsymbol{C} \\
(\star) & \gamma^{2} \overline{\boldsymbol{D}}^{\mathrm{T}} \boldsymbol{M}^{-1} \overline{\boldsymbol{D}}-\epsilon \eta_{3}+4 \sigma\left(\eta_{1}+\eta_{2}\right)
\end{array}\right]<0}
\end{gathered}
$$

are feasible, then the nonlinear dynamics (23) of the tracking error is asymptotically stable.

Proof To demonstrate Theorem 1, we consider first a classical candidate Lyapunov function $V(\overline{\boldsymbol{e}})=\overline{\boldsymbol{e}}^{\mathrm{T}} \boldsymbol{P} \overline{\boldsymbol{e}}$, with $\boldsymbol{P}=\boldsymbol{P}^{\mathrm{T}}$. Then, the tracking error (23) is asymptotically stable if there exists $\boldsymbol{P}$ such that:

$$
\begin{gathered}
V(\overline{\boldsymbol{e}})=\overline{\boldsymbol{e}}^{\mathrm{T}} \boldsymbol{P} \overline{\boldsymbol{e}}>0, \text { such that }-2 \sigma \leq e_{1} \leq 2 \sigma \text { and }\|\overline{\boldsymbol{e}}\|>\epsilon, \\
\dot{V}(\overline{\boldsymbol{e}})=2 \overline{\boldsymbol{e}}^{\mathrm{T}} \boldsymbol{P} \dot{\overline{\boldsymbol{e}}}<0, \text { such that }-2 \sigma \leq e_{1} \leq 2 \sigma \text { and }\|\overline{\boldsymbol{e}}\|>\epsilon,
\end{gathered}
$$

Using expression in (23), it follows that:

$$
\dot{V}(\overline{\boldsymbol{e}})=2 \overline{\boldsymbol{e}}^{\mathrm{T}} \boldsymbol{P}(\boldsymbol{A}+\overline{\boldsymbol{B}} \boldsymbol{K}) \overline{\boldsymbol{e}}+2 \overline{\boldsymbol{e}}^{\mathrm{T}} \boldsymbol{P} \overline{\boldsymbol{D}}\left(f\left(x_{1}\right)-f\left(x_{1}^{r}\right)\right)
$$

Relying on the Young relation (Lemma 1), it yields:

$$
2 \overline{\boldsymbol{e}}^{\mathrm{T}} \boldsymbol{P} \overline{\boldsymbol{D}}\left(f\left(x_{1}\right)-f\left(x_{1}^{r}\right)\right) \leq \overline{\boldsymbol{e}}^{\mathrm{T}} \boldsymbol{P} \boldsymbol{M} \boldsymbol{P} \overline{\boldsymbol{e}}+\overline{\boldsymbol{D}}^{\mathrm{T}} \boldsymbol{M}^{-1} \overline{\boldsymbol{D}}\left(f\left(x_{1}\right)-f\left(x_{1}^{r}\right)\right)^{2}
$$

with $\boldsymbol{M}=\boldsymbol{M}^{\mathrm{T}}>0$.

According to (13), it follows that: $f\left(x_{1}\right)-f\left(x_{1}^{r}\right) \leq \gamma$, with $\gamma=2(\sigma-\sin (\sigma))$. Accordingly, condition (32) can be recast as:

$$
2 \overline{\boldsymbol{e}}^{\mathrm{T}} \boldsymbol{P} \overline{\boldsymbol{D}}\left(\sin \left(x_{1}\right)-\sin \left(x_{1}^{r}\right)\right) \leq \overline{\boldsymbol{e}}^{\mathrm{T}} \boldsymbol{P} \boldsymbol{M} \boldsymbol{P} \overline{\boldsymbol{e}}+\gamma^{2} \overline{\boldsymbol{D}}^{\mathrm{T}} \boldsymbol{M}^{-1} \overline{\boldsymbol{D}}
$$

Hence, using expressions (31) and (33), it follows that:

$$
\dot{V}(\overline{\boldsymbol{e}}) \leq 2 \overline{\boldsymbol{e}}^{\mathrm{T}} \boldsymbol{P}(\boldsymbol{A}+\overline{\boldsymbol{B}} \boldsymbol{K}) \overline{\boldsymbol{e}}+\overline{\boldsymbol{e}}^{\mathrm{T}} \boldsymbol{P} \boldsymbol{M} \boldsymbol{P} \overline{\boldsymbol{e}}+\gamma^{2} \overline{\boldsymbol{D}}^{\mathrm{T}} \boldsymbol{M}^{-1} \overline{\boldsymbol{D}}
$$

Posing $U(\overline{\boldsymbol{e}})=2 \overline{\boldsymbol{e}}^{\mathrm{T}} \boldsymbol{P}(\boldsymbol{A}+\overline{\boldsymbol{B}} \boldsymbol{K}) \overline{\boldsymbol{e}}+\overline{\boldsymbol{e}}^{\mathrm{T}} \boldsymbol{P} \boldsymbol{M} \boldsymbol{P} \overline{\boldsymbol{e}}+\gamma^{2} \overline{\boldsymbol{D}}^{\mathrm{T}} \boldsymbol{M}^{-1} \overline{\boldsymbol{D}}$. Then, the right-hand side in (34) can be rewritten in the following quadratic representation:

$$
U(\overline{\boldsymbol{e}})=\left[\begin{array}{c}
\overline{\boldsymbol{e}} \\
1
\end{array}\right]^{\mathrm{T}}\left[\begin{array}{cc}
\boldsymbol{P} \boldsymbol{A}+\boldsymbol{P} \overline{\boldsymbol{B}} \boldsymbol{K}+(\star)+\boldsymbol{P} \boldsymbol{M P} & \mathcal{O} \\
(\star) & \gamma^{2} \overline{\boldsymbol{D}}^{\mathrm{T}} \boldsymbol{M}^{-1} \overline{\boldsymbol{D}}
\end{array}\right]\left[\begin{array}{c}
\overline{\boldsymbol{e}} \\
1
\end{array}\right]
$$

In addition, the quantity $V(\overline{\boldsymbol{e}})=\overline{\boldsymbol{e}}^{\mathrm{T}} \boldsymbol{P} \overline{\boldsymbol{e}}$ in (30a) can be rewritten in the following form:

$$
V(\overline{\boldsymbol{e}})=\left[\begin{array}{c}
\overline{\boldsymbol{e}} \\
1
\end{array}\right]^{\mathrm{T}}\left[\begin{array}{cc}
\boldsymbol{P} & \mathcal{O} \\
(\star) & 0
\end{array}\right]\left[\begin{array}{l}
\overline{\boldsymbol{e}} \\
1
\end{array}\right]
$$

Furthermore, the two constraints on the Lyapunov function $V(\overline{\boldsymbol{e}})$ in $(30)$ are equivalent to:

$$
\begin{aligned}
& W_{1}(\overline{\boldsymbol{e}})=\left[\begin{array}{l}
\overline{\boldsymbol{e}} \\
1
\end{array}\right]^{\mathrm{T}}\left[\begin{array}{cc}
\mathcal{O} & \boldsymbol{C} \\
(\star) & -4 \sigma
\end{array}\right]\left[\begin{array}{l}
\overline{\boldsymbol{e}} \\
1
\end{array}\right] \leq 0 \\
& W_{2}(\overline{\boldsymbol{e}})=\left[\begin{array}{c}
\overline{\boldsymbol{e}} \\
1
\end{array}\right]^{\mathrm{T}}\left[\begin{array}{cc}
\mathcal{O} & -\boldsymbol{C} \\
(\star) & -4 \sigma
\end{array}\right]\left[\begin{array}{l}
\overline{\boldsymbol{e}} \\
1
\end{array}\right] \leq 0 \\
& W_{3}(\overline{\boldsymbol{e}})=\left[\begin{array}{c}
\overline{\boldsymbol{e}} \\
1
\end{array}\right]^{\mathrm{T}}\left[\begin{array}{cc}
\mathcal{I} & \mathcal{O} \\
(\star) & -\epsilon
\end{array}\right]\left[\begin{array}{l}
\overline{\boldsymbol{e}} \\
1
\end{array}\right] \geq 0
\end{aligned}
$$

where $\boldsymbol{C}^{\mathrm{T}}=\left[\begin{array}{llll}1 & 0 & 0 & 0\end{array}\right]$.

Accordingly, relying on previous relations, the two constrained inequalities in (30) can be recast as: 


$$
\begin{aligned}
& V(\overline{\boldsymbol{e}})>0 \text { such that } W_{1}(\overline{\boldsymbol{e}}) \leq 0 \text { and } W_{2}(\overline{\boldsymbol{e}}) \leq 0 \text { and } W_{3}(\overline{\boldsymbol{e}}) \geq 0, \\
& U(\overline{\boldsymbol{e}})<0 \text { such that } W_{1}(\overline{\boldsymbol{e}}) \leq 0 \text { and } W_{2}(\overline{\boldsymbol{e}}) \leq 0 \text { and } W_{3}(\overline{\boldsymbol{e}}) \geq 0,
\end{aligned}
$$

Using quadratic representation of each term in (38) and applying the S-procedure (Lemma 4), (38a) and (38b) are equivalent to the existence of positive scalar variables $\mu_{1}, \mu_{2}, \mu_{3}, \eta_{1}, \eta_{2}$ and $\eta_{3}$, and a positive matrix $\boldsymbol{M}$ such that the two matrix inequalities in (29) hold. This ends the proof of Theorem 1.

It is worth noting that the matrix inequality (29a) is linear in $\boldsymbol{P}, \mu_{1}, \mu_{2}$ and $\mu_{3}$. However, the second matrix inequality (29b) is bilinear in terms of $\boldsymbol{P}, \boldsymbol{K}, \boldsymbol{M}, \eta_{1}, \eta_{2}$ and $\eta_{3}$. Then, we stress that the stabilization problem of the tracking error is transformed into the solving problem of two BMIs. Here, the LMI (29a) can be considered as a BMI as (29b). However, the BMIs problem in Theorem 1 is nonconvex and difficult to obtain a solution. Therefore, our objective lies in the transformation of these two BMIs (29a) and (29b) into LMIs, which will be used to allow a numerical solution of the stabilization problem. In the sequel, we deal with the linearization of these BMIs.

Remark 5 According to Remark 2, if we consider an underactuated IWIP subject to external disturbances and under parametric uncertainties where its dynamics is described by (17), we will obtain (almost) the same optimization problem in Theorem 1 with the two BMIs (29a) and (29b). In fact, the BMI (29a) is unchanged. However, the BMI (29b) is slightly modified and replaced by the BMI (95) (see Appendix 2 for further details on this obtained BMI).

\subsection{Linearization of the BMIs}

In this part, we deal with the linearization of the two BMIs in (29). Then, we will linearize first the second BMI (29b). The linearization of the first BMI (29a) will be achieved almost with the same method.

\subsubsection{Linearization of the BMI (29b)}

We linearize first the BMI (29b). Posing $\boldsymbol{S}=\boldsymbol{P}^{-1}$. Left and right multiplying expression (29b) by $\left[\begin{array}{cc}\boldsymbol{S} & \mathcal{O} \\ (\star) & 1\end{array}\right]$ yields the following condition

$$
\left[\begin{array}{cc}
\boldsymbol{A} \boldsymbol{S}+\overline{\boldsymbol{B}} \boldsymbol{R}+(\star)+\boldsymbol{M}+\eta_{3} \boldsymbol{S}^{2} & -\left(\eta_{1}-\eta_{2}\right) \boldsymbol{S} \boldsymbol{C} \\
(\star) & \gamma^{2} \overline{\boldsymbol{D}}^{\mathrm{T}} \boldsymbol{M}^{-1} \overline{\boldsymbol{D}}-\epsilon \eta_{3}+4 \sigma\left(\eta_{1}+\eta_{2}\right)
\end{array}\right]<0
$$

with $\boldsymbol{R}=\boldsymbol{K} \boldsymbol{S}$

Relying on the Schur complement, and since $\boldsymbol{M}>0$, it is easy to demonstrate that (39) is equivalent to:

$$
\left[\begin{array}{ccc}
\boldsymbol{A} \boldsymbol{S}+\overline{\boldsymbol{B}} \boldsymbol{R}+(\star)+\boldsymbol{M}+\eta_{3} \boldsymbol{S}^{2} & -\left(\eta_{1}-\eta_{2}\right) \boldsymbol{S} \boldsymbol{C} & \mathcal{O} \\
(\star) & -\epsilon \eta_{3}+4 \sigma\left(\eta_{1}+\eta_{2}\right) & \gamma \overline{\boldsymbol{D}}^{\mathrm{T}} \\
(\star) & (\star) & -\boldsymbol{M}
\end{array}\right]<0
$$

Furthermore, left and right multiplying expression (40) by $\left[\begin{array}{lll}\mathcal{I} & \mathcal{O} & \mathcal{O} \\ \mathcal{O} & \mathcal{O} & \mathcal{I} \\ \mathcal{O} & \mathcal{I} & \mathcal{O}\end{array}\right]$ gives:

$$
\left[\begin{array}{ccc}
\boldsymbol{A} \boldsymbol{S}+\overline{\boldsymbol{B}} \boldsymbol{R}+(\star)+\boldsymbol{M}+\eta_{3} \boldsymbol{S}^{2} & \mathcal{O} & -\left(\eta_{1}-\eta_{2}\right) \boldsymbol{S} \boldsymbol{C} \\
(\star) & -\boldsymbol{M} & \gamma \overline{\boldsymbol{D}} \\
(\star) & (\star) & -\epsilon \eta_{3}+4 \sigma\left(\eta_{1}+\eta_{2}\right)
\end{array}\right]<0
$$

For simplicity, posing $\boldsymbol{X}=\left[\begin{array}{cc}\boldsymbol{A} \boldsymbol{S}+\overline{\boldsymbol{B}} \boldsymbol{R}+(\star)+\boldsymbol{M}+\eta_{3} \boldsymbol{S}^{2} & \mathcal{O} \\ (\star) & -\boldsymbol{M}\end{array}\right], \boldsymbol{Y}=\left[\begin{array}{c}\mathcal{O} \\ \gamma \overline{\boldsymbol{D}}\end{array}\right], \boldsymbol{Z}=\left[\begin{array}{c}-\left(\eta_{1}-\eta_{2}\right) \boldsymbol{S C} \\ \mathcal{O}\end{array}\right]$, and $\varphi_{1}=-\epsilon \eta_{3}+4 \sigma\left(\eta_{1}+\eta_{2}\right)$. Then, inequality (41) can be reformulated like so:

$$
\left[\begin{array}{lc}
\boldsymbol{X} & \boldsymbol{Y}+\boldsymbol{Z} \\
(\star) & \varphi_{1}
\end{array}\right]<0
$$

Applying the Schur complement Lemma yields:

$$
\boldsymbol{X}-(\boldsymbol{Y}+\boldsymbol{Z}) \varphi_{1}^{-1}(\boldsymbol{Y}+\boldsymbol{Z})^{\mathrm{T}}<0
$$




$$
\varphi_{1}<0
$$

Moreover, posing $\boldsymbol{L}=\left[\begin{array}{l}1 \\ 1\end{array}\right], \boldsymbol{N}=\left[\begin{array}{cc}\beta_{1} & 0 \\ 0 & \beta_{2}\end{array}\right], \beta_{1}=\eta_{1}^{-1}, \beta_{2}=\eta_{2}^{-1}, \boldsymbol{Q}^{-1}=4 \sigma \mathcal{I}$ and $\xi_{1}^{-1}=-\epsilon \eta_{3}$, where $\xi_{1}<0$ and $\boldsymbol{N}>0$. Then, we obtain:

$$
\varphi_{1}=\xi_{1}^{-1}+\boldsymbol{L}^{\mathrm{T}} \boldsymbol{N}^{-1} \boldsymbol{Q}^{-1} \boldsymbol{L}
$$

Applying now the matrix inversion lemma to the expression of $\varphi_{1}$ in (44). Hence, it yields:

$$
\varphi_{1}^{-1}=\xi_{1}-\xi_{1}^{2} \boldsymbol{L}^{\mathrm{T}} \boldsymbol{H}^{-1} \boldsymbol{L}
$$

with

$$
\boldsymbol{H}=\xi_{1} \boldsymbol{L} \boldsymbol{L}^{\mathrm{T}}+\boldsymbol{Q} \boldsymbol{N}
$$

In addition, we can show that: $\boldsymbol{Z}=\boldsymbol{G} \boldsymbol{N}^{-1} \boldsymbol{L}$, with $\boldsymbol{G}=\left[\begin{array}{cc}-\boldsymbol{S} \boldsymbol{C} \boldsymbol{C} \\ \boldsymbol{\mathcal { O }} & \mathcal{O}\end{array}\right]$. Then, using expression (45), we can write the following relation:

$$
\boldsymbol{\Phi}=(\boldsymbol{Y}+\boldsymbol{Z}) \varphi_{1}^{-1}(\boldsymbol{Y}+\boldsymbol{Z})^{\mathrm{T}}=\left(\boldsymbol{Y}+\boldsymbol{G} \boldsymbol{N}^{-1} \boldsymbol{L}\right)\left(\xi_{1}-\xi_{1}^{2} \boldsymbol{L}^{\mathrm{T}} \boldsymbol{H}^{-1} \boldsymbol{L}\right)\left(\boldsymbol{Y}+\boldsymbol{G} \boldsymbol{N}^{-1} \boldsymbol{L}\right)^{\mathrm{T}}
$$

By developing the right-hand side of relation (47), we obtain:

$$
\begin{aligned}
\boldsymbol{\Phi}= & \xi_{1} \boldsymbol{Y} \boldsymbol{Y}^{\mathrm{T}}+\xi_{1} \boldsymbol{Y} \boldsymbol{L}^{\mathrm{T}} \boldsymbol{N}^{-1} \boldsymbol{G}^{\mathrm{T}}+\xi_{1} \boldsymbol{G} \boldsymbol{N}^{-1} \boldsymbol{L} \boldsymbol{Y}^{\mathrm{T}}+\xi_{1} \boldsymbol{G} \boldsymbol{N}^{-1} \boldsymbol{L} \boldsymbol{L}^{\mathrm{T}} \boldsymbol{N}^{-1} \boldsymbol{G}^{\mathrm{T}}-\xi_{1}^{2} \boldsymbol{Y} \boldsymbol{L}^{\mathrm{T}} \boldsymbol{H}^{-1} \boldsymbol{L} \boldsymbol{Y}^{\mathrm{T}}- \\
& \xi_{1}^{2} \boldsymbol{Y} \boldsymbol{L}^{\mathrm{T}} \boldsymbol{H}^{-1} \boldsymbol{L} \boldsymbol{L}^{\mathrm{T}} \boldsymbol{N}^{-1} \boldsymbol{G}^{\mathrm{T}}-\xi_{1}^{2} \boldsymbol{G} \boldsymbol{N}^{-1} \boldsymbol{L} \boldsymbol{L}^{\mathrm{T}} \boldsymbol{H}^{-1} \boldsymbol{L} \boldsymbol{Y}^{\mathrm{T}}-\xi_{1}^{2} \boldsymbol{G} \boldsymbol{N}^{-1} \boldsymbol{L} \boldsymbol{L}^{\mathrm{T}} \boldsymbol{H}^{-1} \boldsymbol{L} \boldsymbol{L}^{\mathrm{T}} \boldsymbol{N}^{-1} \boldsymbol{G}^{\mathrm{T}}
\end{aligned}
$$

Moreover, using expression (46) of $\boldsymbol{H}$, it easy to show that:

$$
\begin{gathered}
\xi_{1} \boldsymbol{G} \boldsymbol{N}^{-1} \boldsymbol{L} \boldsymbol{L}^{\mathrm{T}} \boldsymbol{N}^{-1} \boldsymbol{G}^{\mathrm{T}}=\boldsymbol{G} \boldsymbol{N}^{-1} \boldsymbol{H} \boldsymbol{N}^{-1} \boldsymbol{G}^{\mathrm{T}}-\boldsymbol{G} \boldsymbol{Q} \boldsymbol{N}^{-1} \boldsymbol{G}^{\mathrm{T}} \\
\xi_{1}^{2} \boldsymbol{G} \boldsymbol{N}^{-1} \boldsymbol{L} \boldsymbol{L}^{\mathrm{T}} \boldsymbol{H}^{-1} \boldsymbol{L} \boldsymbol{L}^{\mathrm{T}} \boldsymbol{N}^{-1} \boldsymbol{G}^{\mathrm{T}}=\boldsymbol{G} \boldsymbol{N}^{-1} \boldsymbol{H} \boldsymbol{N}^{-1} \boldsymbol{G}^{\mathrm{T}}-2 \boldsymbol{G} \boldsymbol{Q} \boldsymbol{N}^{-1} \boldsymbol{G}^{\mathrm{T}}+\boldsymbol{G} \boldsymbol{Q} \boldsymbol{H}^{-1} \boldsymbol{Q} \boldsymbol{G}^{\mathrm{T}} \\
\xi_{1}^{2} \boldsymbol{G} \boldsymbol{N}^{-1} \boldsymbol{L} \boldsymbol{L}^{\mathrm{T}} \boldsymbol{H}^{-1} \boldsymbol{L} \boldsymbol{Y}^{\mathrm{T}}=\xi_{1} \boldsymbol{G} \boldsymbol{N}^{-1} \boldsymbol{L} \boldsymbol{Y}^{\mathrm{T}}-\xi_{1} \boldsymbol{G} \boldsymbol{Q} \boldsymbol{H}^{-1} \boldsymbol{L} \boldsymbol{Y}^{\mathrm{T}}
\end{gathered}
$$

Then, using expressions in (49), relation (48) can be simplified like so:

$$
\begin{aligned}
\boldsymbol{\Phi}= & \xi_{1} \boldsymbol{Y} \boldsymbol{Y}^{\mathrm{T}}-\xi_{1}^{2} \boldsymbol{Y} \boldsymbol{L}^{\mathrm{T}} \boldsymbol{H}^{-1} \boldsymbol{L} \boldsymbol{Y}^{\mathrm{T}}+\xi_{1} \boldsymbol{G} \boldsymbol{Q} \boldsymbol{H}^{-1} \boldsymbol{L} \boldsymbol{Y}^{\mathrm{T}}+ \\
& \xi_{1} \boldsymbol{Y} \boldsymbol{L}^{\mathrm{T}} \boldsymbol{H}^{-1} \boldsymbol{Q} \boldsymbol{G}^{\mathrm{T}}+\boldsymbol{G} \boldsymbol{Q} \boldsymbol{N}^{-1} \boldsymbol{G}^{\mathrm{T}}-\boldsymbol{G} \boldsymbol{Q} \boldsymbol{H}^{-1} \boldsymbol{Q} \boldsymbol{G}^{\mathrm{T}}
\end{aligned}
$$

This expression (50) can be rewritten as follows:

$$
\boldsymbol{\Phi}=\xi_{1} \boldsymbol{Y} \boldsymbol{Y}^{\mathrm{T}}-\left(\xi_{1} \boldsymbol{Y} \boldsymbol{L}^{\mathrm{T}}-\boldsymbol{Q} \boldsymbol{G}\right) \boldsymbol{H}^{-1}\left(\xi_{1} \boldsymbol{Y} \boldsymbol{L}^{\mathrm{T}}-\boldsymbol{Q} \boldsymbol{G}\right)^{\mathrm{T}}+\boldsymbol{G} \boldsymbol{Q} \boldsymbol{N}^{-1} \boldsymbol{G}^{\mathrm{T}}
$$

Therefore, substituting expression of $\boldsymbol{\Phi}=(\boldsymbol{Y}+\boldsymbol{Z}) \varphi_{1}^{-1}(\boldsymbol{Y}+\boldsymbol{Z})^{\mathrm{T}}$ in the inequality (43a) yields:

$$
\boldsymbol{X}-\xi_{1} \boldsymbol{Y} \boldsymbol{Y}^{\mathrm{T}}+\left(\xi_{1} \boldsymbol{Y} \boldsymbol{L}^{\mathrm{T}}-\boldsymbol{Q} \boldsymbol{G}\right) \boldsymbol{H}^{-1}\left(\xi_{1} \boldsymbol{Y} \boldsymbol{L}^{\mathrm{T}}-\boldsymbol{Q} \boldsymbol{G}\right)^{\mathrm{T}}-\boldsymbol{G} \boldsymbol{Q} \boldsymbol{N}^{-1} \boldsymbol{G}^{\mathrm{T}}<0
$$

Since $\boldsymbol{N}>0$, then inequality (52) can be simplified as:

$$
\boldsymbol{X}-\xi_{1} \boldsymbol{Y} \boldsymbol{Y}^{\mathrm{T}}+\left(\xi_{1} \boldsymbol{Y} \boldsymbol{L}^{\mathrm{T}}-\boldsymbol{Q} \boldsymbol{G}\right) \boldsymbol{H}^{-1}\left(\xi_{1} \boldsymbol{Y} \boldsymbol{L}^{\mathrm{T}}-\boldsymbol{Q} \boldsymbol{G}\right)^{\mathrm{T}}<0
$$

Moreover, as $\xi_{1}<0, \boldsymbol{L} \boldsymbol{L}^{\mathrm{T}} \geq 0$ and $\boldsymbol{N}>0$, then relying on expression (46), we can obtain $\boldsymbol{H}>0$. Accordingly, based on the Schur complement, the inequality (53) is equivalent to:

$$
\left[\begin{array}{cc}
\boldsymbol{X}-\xi_{1} \boldsymbol{Y} \boldsymbol{Y}^{\mathrm{T}} & \xi_{1} \boldsymbol{Y} \boldsymbol{L}^{\mathrm{T}}-\boldsymbol{Q} \boldsymbol{G} \\
(\star) & -\boldsymbol{H}
\end{array}\right]<0
$$

Substituting expressions of $\boldsymbol{X}, \boldsymbol{Y}, \boldsymbol{G}, \boldsymbol{L}, \boldsymbol{Q}, \boldsymbol{H}$ in (54), and using the Schur complement for the quantity $\eta_{3} S^{2}$, and as $\xi_{1}^{-1}=-\epsilon \eta_{3}<0$, we obtain then the following LMI: 


$$
\left[\begin{array}{ccccc}
\boldsymbol{A} \boldsymbol{S}+\overline{\boldsymbol{B}} \boldsymbol{R}+(\star)+\boldsymbol{M} & \mathcal{O} & \frac{1}{4 \sigma} \boldsymbol{S C} & -\frac{1}{4 \sigma} \boldsymbol{S C} & \boldsymbol{S} \\
(\star) & -\boldsymbol{M}-\xi_{1} \gamma^{2} \overline{\bar{D}} \overline{\boldsymbol{D}}^{\mathrm{T}} & \xi_{1} \gamma \overline{\boldsymbol{D}} & \xi_{1} \gamma \overline{\boldsymbol{D}} & \mathcal{O} \\
(\star) & (\star) & -\frac{1}{4 \sigma} \beta_{1}-\xi_{1} & -\xi_{1} & \mathcal{O} \\
(\star) & (\star) & (\star) & -\frac{1}{4 \sigma} \beta_{2}-\xi_{1} & \mathcal{O} \\
(\star) & (\star) & (\star) & (\star) & \epsilon \xi_{1} \mathcal{I}
\end{array}\right]<0
$$

Moreover, using expression (44) in (43b) and multiplying it by the quantity $\xi_{1}^{2}$, we obtain the following inequality:

$$
\xi_{1}+\xi_{1}^{2} \boldsymbol{L}^{\mathrm{T}} \boldsymbol{N}^{-1} \boldsymbol{Q}^{-1} \boldsymbol{L}<0
$$

As $\xi_{1}<0$ and $\boldsymbol{N}>0$, then based on the Schur complement, expression (56) is equivalent to:

$$
\left[\begin{array}{cc}
\xi_{1} & \xi_{1} \boldsymbol{L}^{\mathrm{T}} \\
(\star) & -\boldsymbol{Q} \boldsymbol{N}
\end{array}\right]<0
$$

Therefore, we obtain the following LMI:

$$
\left[\begin{array}{ccc}
\xi_{1} & \xi_{1} & \xi_{1} \\
(\star) & -\frac{\beta_{1}}{4 \sigma} & 0 \\
(\star) & (\star) & -\frac{\beta_{2}}{4 \sigma}
\end{array}\right]<0
$$

Hence, the two inequalities (43a) and (43b) are transformed into the two matrix inequalities (55) and (58), respectively.

\subsubsection{Linearization of the BMI (29a)}

Linearization of the BMI (29a) is almost identical to that of the BMI (29b). As $\boldsymbol{S}=\boldsymbol{P}^{-1}$, and left and right multiplying expression $(29 a)$ by the matrix $\left[\begin{array}{cc}\boldsymbol{S} & \mathcal{O} \\ (\star & 1\end{array}\right]$, we will obtain then the following result:

$$
\left[\begin{array}{cc}
\boldsymbol{S}-\mu_{3} \boldsymbol{S}^{2} & \left(\mu_{1}-\mu_{2}\right) \boldsymbol{S} \boldsymbol{C} \\
(\star) & \epsilon \mu_{3}-4 \sigma\left(\mu_{1}+\mu_{2}\right)
\end{array}\right]>0
$$

Let us pose $\varphi_{2}=\epsilon \mu_{3}-4 \sigma\left(\mu_{1}+\mu_{2}\right), \boldsymbol{N}=\left[\begin{array}{cc}\alpha_{1} & 0 \\ 0 & \alpha_{2}\end{array}\right], \alpha_{1}=\mu_{1}^{-1}, \alpha_{2}=\mu_{2}^{-1}$, and $\xi_{2}^{-1}=\epsilon \mu_{3}$, where $\xi_{2}>0$ and $N>0$. Then, we obtain:

$$
\varphi_{2}=\xi_{2}^{-1}-\boldsymbol{L}^{\mathrm{T}} \boldsymbol{N}^{-1} \boldsymbol{Q}^{-1} \boldsymbol{L}
$$

Moreover, we can show that: $\left(\mu_{1}-\mu_{2}\right) \boldsymbol{S C}=-\boldsymbol{G} \boldsymbol{N}^{-1} \boldsymbol{L}$.

Based on the Schur complement, the matrix inequality (59) is equivalent to:

$$
\begin{gathered}
\boldsymbol{S}-\left(\epsilon \xi_{2}\right)^{-1} \boldsymbol{S}^{2}-\boldsymbol{G} \boldsymbol{N}^{-1} \boldsymbol{L} \varphi_{2}^{-1} \boldsymbol{L}^{\mathrm{T}} \boldsymbol{N}^{-1} \boldsymbol{G}^{\mathrm{T}}>0 \\
\varphi_{2}>0
\end{gathered}
$$

The matrix inversion lemma states that:

$$
\varphi_{2}^{-1}=\xi_{2}-\xi_{2}^{2} \boldsymbol{L}^{\mathrm{T}} \boldsymbol{H}^{-1} \boldsymbol{L}
$$

with

$$
\boldsymbol{H}=\xi_{2} \boldsymbol{L} \boldsymbol{L}^{\mathrm{T}}-\boldsymbol{Q} \boldsymbol{N}
$$

Substituting expression (62) in (61a) yields:

$$
\boldsymbol{S}-\left(\epsilon \xi_{2}\right)^{-1} \boldsymbol{S}^{2}-\xi_{2} \boldsymbol{G} \boldsymbol{N}^{-1} \boldsymbol{L} \boldsymbol{L}^{\mathrm{T}} \boldsymbol{N}^{-1} \boldsymbol{G}^{\mathrm{T}}+\xi_{2}^{2} \boldsymbol{G} \boldsymbol{N}^{-1} \boldsymbol{L} \boldsymbol{L}^{\mathrm{T}} \boldsymbol{H}^{-1} \boldsymbol{L} \boldsymbol{L}^{\mathrm{T}} \boldsymbol{N}^{-1} \boldsymbol{G}^{\mathrm{T}}>0
$$

Based on expression of $\boldsymbol{H}$ in (63), we can show that:

$$
\begin{gathered}
\xi_{2} \boldsymbol{G} \boldsymbol{N}^{-1} \boldsymbol{L} \boldsymbol{L}^{\mathrm{T}} \boldsymbol{N}^{-1} \boldsymbol{G}^{\mathrm{T}}=\boldsymbol{G} \boldsymbol{N}^{-1} \boldsymbol{H} \boldsymbol{N}^{-1} \boldsymbol{G}^{\mathrm{T}}+\boldsymbol{G} \boldsymbol{Q} \boldsymbol{N}^{-1} \boldsymbol{G}^{\mathrm{T}} \\
\xi_{1}^{2} \boldsymbol{G} \boldsymbol{N}^{-1} \boldsymbol{L} \boldsymbol{L}^{\mathrm{T}} \boldsymbol{H}^{-1} \boldsymbol{L} \boldsymbol{L}^{\mathrm{T}} \boldsymbol{N}^{-1} \boldsymbol{G}^{\mathrm{T}}=\boldsymbol{G} \boldsymbol{N}^{-1} \boldsymbol{H} \boldsymbol{N}^{-1} \boldsymbol{G}^{\mathrm{T}}+2 \boldsymbol{G} \boldsymbol{Q} \boldsymbol{N}^{-1} \boldsymbol{G}^{\mathrm{T}}+\boldsymbol{G} \boldsymbol{Q} \boldsymbol{H}^{-1} \boldsymbol{Q} \boldsymbol{G}^{\mathrm{T}}
\end{gathered}
$$

Substituting these two expressions into (63) leads to a simplified inequality: 


$$
\boldsymbol{S}-\left(\epsilon \xi_{2}\right)^{-1} \boldsymbol{S}^{2}+\boldsymbol{G} \boldsymbol{Q} \boldsymbol{N}^{-1} \boldsymbol{G}^{\mathrm{T}}+\boldsymbol{G} \boldsymbol{Q} \boldsymbol{H}^{-1} \boldsymbol{Q} \boldsymbol{G}^{\mathrm{T}}>0
$$

As $\boldsymbol{N}>0$, it follows that inequality (66) can be recast as:

$$
\boldsymbol{S}-\left(\epsilon \xi_{2}\right)^{-1} \boldsymbol{S}^{2}+\boldsymbol{G} \boldsymbol{Q} \boldsymbol{H}^{-1} \boldsymbol{Q} \boldsymbol{G}^{\mathrm{T}}>0
$$

In addition, according to expression (63) and as we can have $\boldsymbol{H}<0$, the Schur complement states that the matrix inequality (67) is equivalent to:

$$
\left[\begin{array}{ccc}
\boldsymbol{S} & \boldsymbol{Q G} & \boldsymbol{S} \\
(\star) & -\boldsymbol{H} & \mathcal{O} \\
(\star) & (\star) & \epsilon \xi_{2} \mathcal{I}
\end{array}\right]>0
$$

Accordingly, we obtain the following LMI:

$$
\left[\begin{array}{cccc}
\boldsymbol{S} & -\frac{1}{4 \sigma} \boldsymbol{S} & \frac{1}{4 \sigma} \boldsymbol{S} \boldsymbol{C} & \boldsymbol{S} \\
(\star) & \frac{1}{4 \sigma} \alpha_{1}-\xi_{2} & -\xi_{2} & \mathcal{O} \\
(\star) & (\star) & \frac{1}{4 \sigma} \alpha_{2}-\xi_{2} & \mathcal{O} \\
(\star) & (\star) & (\star) & \epsilon \xi_{2} \mathcal{I}
\end{array}\right]>0
$$

Furthermore, relying on the previous subsection, we can show that the condition (61b) is equivalent to:

$$
\left[\begin{array}{ccc}
\xi_{2} & \xi_{2} & \xi_{2} \\
(\star) & \frac{\alpha_{1}}{4 \sigma} & 0 \\
(\star) & (\star) & \frac{\alpha_{2}}{4 \sigma}
\end{array}\right]>0
$$

Then, the two inequalities (61a) and (61b) are reformulated by the two matrix inequalities (69) and (70), respectively.

Hence, relying on previous results, we can state the following theorem.

Theorem 2 If there exist a symmetric matrix $\boldsymbol{S}$, a matrix $\boldsymbol{R}$, a positive-definite symmetric matrix $\boldsymbol{M}$, some constants $\alpha_{1}>0, \alpha_{2}>0, \beta_{1}>0, \beta_{2}>0, \xi_{1}<0$ and $\xi_{2}>0$, such that the following LMIs

$$
\begin{aligned}
& {\left[\begin{array}{ccc}
\xi_{2} & \xi_{2} & \xi_{2} \\
(\star) & \frac{\alpha_{1}}{4 \sigma} & 0 \\
(\star) & (\star) & \frac{\alpha_{2}}{4 \sigma}
\end{array}\right]>0} \\
& {\left[\begin{array}{ccc}
\xi_{1} & \xi_{1} & \xi_{1} \\
(\star) & -\frac{\beta_{1}}{4 \sigma} & 0 \\
(\star) & (\star) & -\frac{\beta_{2}}{4 \sigma}
\end{array}\right]<0} \\
& {\left[\begin{array}{cccc}
\boldsymbol{S} & -\frac{1}{4 \sigma} \boldsymbol{S C} & \frac{1}{4 \sigma} \boldsymbol{S C} & \boldsymbol{S} \\
(\star) & \frac{1}{4 \sigma} \alpha_{1}-\xi_{2} & -\xi_{2} & \mathcal{O} \\
(\star) & (\star) & \frac{1}{4 \sigma} \alpha_{2}-\xi_{2} & \mathcal{O} \\
(\star) & (\star) & (\star) & \epsilon \xi_{2} \mathcal{I}
\end{array}\right]>0} \\
& {\left[\begin{array}{ccccc}
\boldsymbol{A} \boldsymbol{S}+\overline{\boldsymbol{B}} \boldsymbol{R}+(\star)+\boldsymbol{M} & \mathcal{O} & \frac{1}{4 \sigma} \boldsymbol{S C} & -\frac{1}{4 \sigma} \boldsymbol{S C} & \boldsymbol{S} \\
(\star) & -\boldsymbol{M}-\xi_{1} \gamma^{2} \overline{\boldsymbol{D}} \overline{\boldsymbol{D}}^{\mathrm{T}} & \xi_{1} \gamma \overline{\boldsymbol{D}} & \xi_{1} \gamma \overline{\boldsymbol{D}} & \mathcal{O} \\
(\star) & (\star) & -\frac{1}{4 \sigma} \beta_{1}-\xi_{1} & -\xi_{1} & \mathcal{O} \\
(\star) & (\star) & (\star) & -\frac{1}{4 \sigma} \beta_{2}-\xi_{1} & \mathcal{O} \\
(\star) & (\star) & (\star) & (\star) & \epsilon \xi_{1} \mathcal{I}
\end{array}\right]<0}
\end{aligned}
$$

are feasible, then the nonlinear dynamics (18) of the tracking error is asymptotically stable. In addition, the gain matrix of the control law (22) is defined by: $\boldsymbol{K}=\boldsymbol{R} \boldsymbol{S}^{-1}$.

It is worth mentioning that in Theorem 2, the stability conditions of the nonlinear system (18) are reformulated by four LMIs (71a)-(71d). These LMIs are defined with respect to the unknown matrices $\boldsymbol{S}, \boldsymbol{R}$ and $\boldsymbol{M}$, and unknown variables $\xi_{1}, \xi_{2}, \alpha_{1}, \alpha_{2}, \beta_{1}$ and $\beta_{2}$. Then, the problem lies in finding such unknown matrices and variables satisfying the four LMIs in Theorem 2, which can be easily and effectively solved numerically via LMI control toolbox [87]. Once the solving problem is feasible, then the gain matrix $\boldsymbol{K}$ is computed by means of relation: $K=R \boldsymbol{S}^{-1}$. 


\section{Simulation results of the limit cycle tracking}

Simulation results are presented and discussed in the following. They attest to the effectiveness of the designed control law (20) for the tracking of the self-generated period-1 stable limit cycle using the gain matrix $\boldsymbol{K}$, which is solution of LMIs in Theorem 2. We recall that the matrices $\boldsymbol{A}, \overline{\boldsymbol{B}}, \overline{\boldsymbol{D}}$ and $\boldsymbol{C}$ are given in the beginning of Section 4. Moreover, $\sigma=10^{\circ}$. Thus, by choosing $\epsilon=0.1$, the resolution of the four LMIs in (71) by means of the LMI toolbox of MATLAB gives the following results: $\boldsymbol{S}=\left[\begin{array}{ccc}49.5559 & -16.3793 & -10.6745 \\ -16.3793 & 20.9659 & -20.6407 \\ -10.6745 & -20.6407 & 50.1719\end{array}\right]$, $\boldsymbol{M}=\left[\begin{array}{ccc}398.7800 & -21.5710 & -21.5706 \\ -21.5710 & 324.4302 & -298.2469 \\ -21.5706 & -298.2469 & 324.5022\end{array}\right], \boldsymbol{R}=[1088.26171 .7705-844.0613], \xi_{1}=-570.8652, \xi_{2}=886.3538$, $\alpha_{1}=\alpha_{2}=1497.858$, and $\beta_{1}=\beta_{2}=1070.568$. Therefore, the control gain $\boldsymbol{K}$ is computed to be: $\boldsymbol{K}=$ [48.5437 66.7622 20.9706].

Table 2 shows some numerical values of the matrix gain $\boldsymbol{K}$ for some values of the parameter $\epsilon$. We emphasize that as $\epsilon$ decreases, the gain $\boldsymbol{K}$ increases. This increase in $\boldsymbol{K}$ will provoke a considerable increase in the control effort. However, the tracking error will be canceled rapidly as $\boldsymbol{K}$ increases. This will be shown next.

Table 2 Numerical values of the gain matrix $\boldsymbol{K}$ for different values of $\epsilon$.

\begin{tabular}{|c|c|c|c|}
\hline$\epsilon$ & & $K$ & \\
\hline 0.1 & 48.5437 & 66.7622 & 20.9706 \\
\hline 0.05 & 304.4274 & 603.7444 & 298.4429 \\
\hline 0.01 & 239.0992 & 500.4151 & 196.2109 \\
\hline 0.005 & 343.4758 & 786.0560 & 290.5334 \\
\hline 0.001 & 4768.16 & 10418.68 & 4837.79 \\
\hline 0.0005 & 3230.59 & 7633.10 & 2909.52 \\
\hline
\end{tabular}

We recall that $\boldsymbol{K}$ is the gain matrix of the stabilizing control law $w$ defined by expression (22). Moreover, it is worth noting that the tracking control law (20) can be rewritten as:

$$
v=\hat{\boldsymbol{K}} \boldsymbol{e}=\hat{\boldsymbol{K}}\left(\boldsymbol{x}-\boldsymbol{x}^{r}\right)
$$

with $\hat{\boldsymbol{K}}=\left[\boldsymbol{K}(1)-k_{2} \boldsymbol{K}(2) \boldsymbol{K}(3)\right]$, where $\boldsymbol{K}(i)$ is the $i$ th element of the gain matrix $\boldsymbol{K}$.

Furthermore, in order to avoid high gain of the tracking control law $v$ in (72), we use a saturation function as follows:

$$
v=\left\{\begin{array}{llr}
v_{\max } & \text { if } & v>v_{\max } \\
\hat{\boldsymbol{K}}\left(\boldsymbol{x}-\boldsymbol{x}^{r}\right) & \text { if }-v_{\max } \leq v \leq v_{\max } \\
-v_{\max } & \text { if } & v<-v_{\max }
\end{array}\right.
$$

where $v_{\max }=10$. Here, this value of $v_{\max }$ is an arbitrary choice.

We recall that $\boldsymbol{x}^{r}$ is the state of the reference model (16), which generates the desired period-1 stable limit cycle, whereas $\boldsymbol{x}$ is the state of the IWIP, as the physical system modeled via the nonlinear dynamics (15), under the tracking control law $v(73)$.

Our objective is to investigate the influence of the designed control law $v$ with the gain $\boldsymbol{K}$ on the dynamic behavior of the controlled IWIP and then the tracking of the self-generated period-1 stable limit cycle. Moreover, we will consider three different scenarios. The first scenario is the nominal case, for which the system is without parametric uncertainties and without external disturbing torque. In the second scenario, we will take into account uncertain parameters in the dynamics of the controlled IWIP. However, in the third scenario, we will consider the external disturbance. We recall that the dynamics of the IWIP under parametric uncertainties in the friction coefficients $\delta_{1}$ and $\delta_{2}$ and subject to the external disturbing torque $\zeta$ is given by (17). The reference model generating the desired period-1 stable limit cycle is always the nominal one given by the dynamics (16). Moreover, in order to show how well behaved the closed-loop system is to the uncertainties in the two friction parameters $\delta_{1}$ and $\delta_{2}$ and the external disturbance $\zeta$, we will realize several simulations. Indeed, we will consider and analyze different cases: constant uncertain friction parameters, randomly time-varying uncertain parameters, a constant external disturbance, and a randomly time-varying external disturbance. 
As noted previously, it was assumed that the physical device of the IWIP was mechanically designed in order to minimize the effects of viscous frictions [31, 34, 35, 53]. Thus, the frictions of the active and passive joints are completely neglected. Authors in [44] calculated the viscous friction coefficient affecting the actuator, the active joint. It was found to be about $10^{-4}$. In the next, we take a common value for the two friction parameters $\delta_{1}$ and $\delta_{2}, \delta_{1}=\delta_{2}=10^{-2}$.

\subsection{First scenario: Nominal dynamics of the IWIP}

In this first scenario, the IWIP is without uncertainties and external disturbances. As in [53], the proposed simulation is started from the initial condition $\left(\theta_{1}(0), \theta_{2}(0), \dot{\theta}_{1}(0), \dot{\theta}_{2}(0)\right)=\left(10^{\circ}, 0^{\circ}, 0 \mathrm{rad} / \mathrm{s}, 0 \mathrm{rad} / \mathrm{s}\right)$. The choice of $\theta_{1}(0)$ is motivated by the physical properties of the IWIP since simulation is started from the rest position at standstill. Moreover, we will take the gain matrix $\boldsymbol{K}$ computed for the parameter $\epsilon=0.1$ (see Table 2).

Then, application of the control law (73) to the nominal nonlinear dynamics (15) of IWIP gives the results in Fig. 6. In each portrait of Fig. 6, we show the variable of the reference model and that of the controlled IWIP system, which is given by (15). Figure 6(a) shows the temporal evolution of the angular position of the pendulum body, $\theta_{1}$. Figure $6(\mathrm{~b})$ reveals the angular velocity of the pendulum $\dot{\theta}_{1}$. Figure $6(\mathrm{c})$ depicts the angular velocity of the inertia wheel $\dot{\theta}_{2}$. Figure $6(\mathrm{~d})$ shows the desired period-1 stable limit cycle and the trajectory of the IWIP system under the control law (73) from the proposed initial condition. These results show clearly the convergence of the trajectory of the controlled IWIP to the reference trajectory and hence to the desired stable period-1 limit cycle. It can be observed from the displayed results that the tracking was achieved within almost three periods, since the period of the reference limit cycle is about $0.6[s]$. Figure. 7 represents the tracking control law $v$. When the tracking was reached, the control law $v$ remains constant about the value $-0.0614[N]$. It is clear that the control effort is initially saturated at the value $10[N]$.

We have chosen another initial condition for the controlled IWIP such that $\theta_{1}(0)$ is away from the tracking trajectory. Then, using the same matrix gain $\boldsymbol{K}$ and the initial condition $\left(\theta_{1}(0), \theta_{2}(0), \dot{\theta}_{1}(0), \dot{\theta}_{2}(0)\right)=$ $\left(0^{\circ}, 0^{\circ}, 0 \mathrm{rad} / \mathrm{s}, 0 \mathrm{rad} / \mathrm{s}\right)$, we obtained the simulation results in Fig. 8. Figure 8(a) shows the trajectory of the controlled IWIP in the state space with the desired period-1 stable limit cycle. Figure 8(b) shows temporal evolution of the tracking control law $v$.

In the two last simulation results, the initial condition was selected to respect the unilateral state constraints of the inverted pendulum given by (1). We have chosen other initial positions of the inverted pendulum such that the angular position $\theta_{1}$ is outside the interval $\left[\begin{array}{cc}-\sigma & \sigma\end{array}\right]$. In this case, the physical device of the underactuated IWIP is not that given in Fig. 1. The IWIP is not under the unilateral constraints and its swing motion is completely free. In this case, we will have the inertial wheel pendulum studied by Aguilar and his co-workers (see for example [45]) and other authors with different physical parameters. Figure 9 reveals simulation results of the underactuated IWIP for two initial positions of the inverted pendulum lying outside the defined working space (1). Figure 9(a) and Fig. 9(c) show the trajectory of the inverted pendulum in the state space and the corresponding self-generated limit cycle. However, Fig. 9(b) and Fig. 9(d) represent the tracking control law v. Figure 9(a) and Fig. 9(b) are plotted for the initial condition $\theta_{1}(0)=90^{\circ}$, whereas Fig. $9(\mathrm{c})$ and Fig. $9(\mathrm{~d})$ are depicted for $\theta_{1}(0)=180^{\circ}$. It is obvious that in these two cases the trajectory of the IWIP follows the desired period- 1 stable limit cycle. Moreover, the control law $v$ tends to a very small value (almost zero). It is worth noting that initially the control law $v$ is saturated at $\pm 10[N]$. This is because the IWIP needs an important control effort to bring the IWIP from its initial position to the desired one where the initial position was chosen to be far away from the tracking trajectory.

\subsection{Second scenario: IWIP under uncertain friction parameters}

As noted before, in this second scenario, the two friction coefficients $\delta_{1}$ and $\delta_{2}$ of the passive and the active articulations of the controlled underactuated IWIP are considered to be uncertain. Moreover, we will assume that $0 \leq \delta_{1}, \delta_{2} \leq 10^{-3}$. In this analysis, the initial condition of the IWIP is $\left(\theta_{1}(0), \theta_{2}(0), \dot{\theta}_{1}(0), \dot{\theta}_{2}(0)\right)=$ $\left(10^{\circ}, 0^{\circ}, 0 \mathrm{rad} / \mathrm{s}, 0 \mathrm{rad} / \mathrm{s}\right)$. Furthermore, the gain matrix $\boldsymbol{K}$ is that calculated for the parameter $\epsilon=0.005$ in Table 2 .

Figs. 10(a)-10(c) show simulation results for the case $\delta_{1}=\delta_{2}=10^{-3}$. In Fig. 10(a) and Fig. 10(b), we plotted the tracking errors $e_{1}$ and $e_{4}$, respectively. Figure 10(c) represents the signal of the control law $v$. It is obvious that the tracking error $e_{1}$ of the inverted pendulum is almost zero $\left(\left|e_{1}\right|<0.04^{\circ}\right)$. Moreover, the 


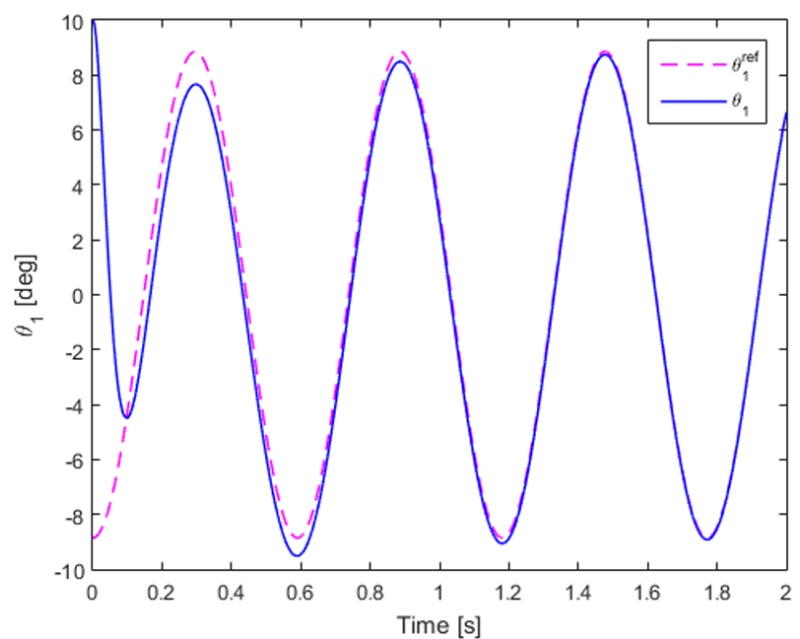

(a)

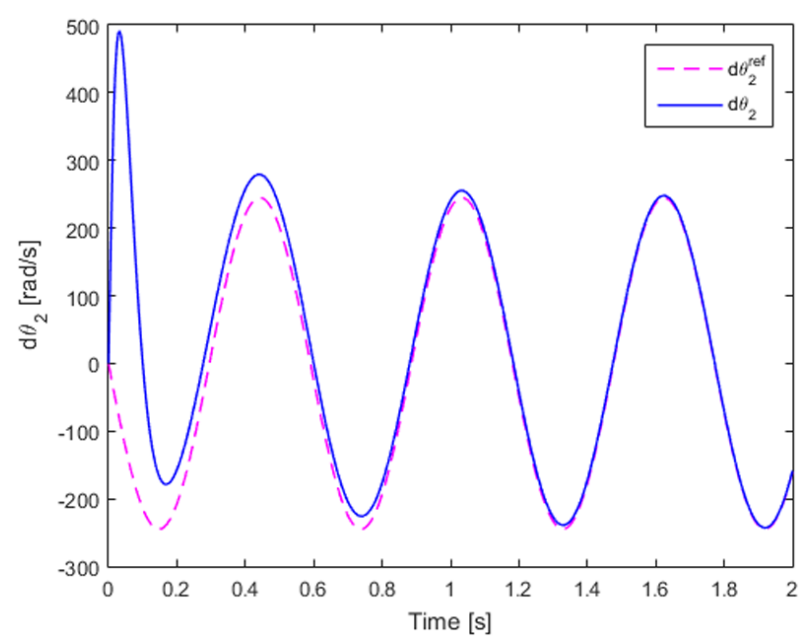

(c)

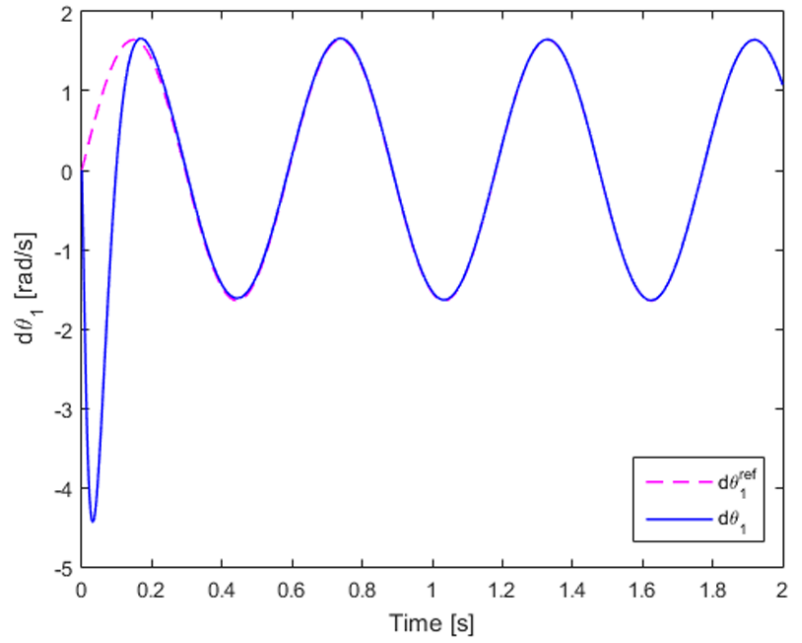

(b)

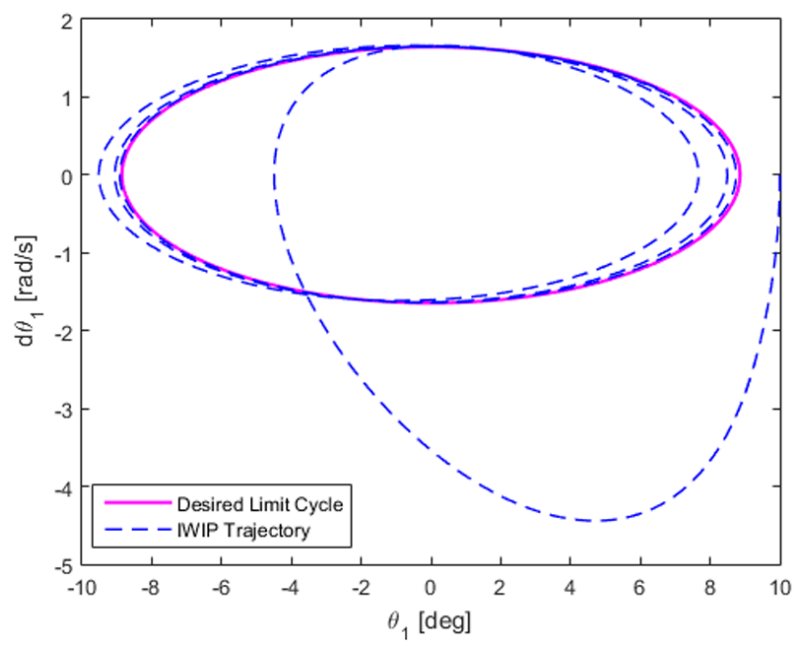

(d)

Fig. 6 Simulation results of the self-generated limit cycle tracking in the nominal case (i.e. the underactuated IWIP is without parametric uncertainties and external disturbance). Here, the IWIP was emanated from the initial condition $\left(\theta_{1}(0), \theta_{2}(0), \dot{\theta}_{1}(0), \dot{\theta}_{2}(0)\right)=\left(10^{\circ}, 0^{\circ}, 0 \mathrm{rad} / \mathrm{s}, 0 \mathrm{rad} / \mathrm{s}\right)$.

tracking error $e_{4}$ is also almost zero, $\left|e_{4}\right|<2[\mathrm{rad} / \mathrm{s}]$. In addition, the control signal $v$ looks like a sinusoidal signal with a weak amplitude about $0.25[N]$.

As the two friction coefficients $\delta_{1}$ and $\delta_{2}$ are unknown, then we have considered them as uncertain parameters and randomly time-varying such that $0 \leq \delta_{1}, \delta_{2} \leq 10^{-3}$. In this case, we used the random function of MATLAB to generate the uncertainties. Figs. 10(d)-10(f) show the corresponding simulation results. As in the previous case, the tracking errors are nearly zero and the control effort is very small.

We stress that the control gain $\boldsymbol{K}$ chosen for the parameter $\epsilon=0.005$ in the present simulation is the ideal one. Indeed, for $\epsilon<0.005$ and using the corresponding gain matrix $\boldsymbol{K}$, the tracking error will increase as $\epsilon$ decreases. For example, for $\epsilon=0.1$, the tracking error $\left|e_{1}\right|<0.3^{\circ}$, whereas $\left|e_{4}\right|<8[\mathrm{rad} / \mathrm{s}]$. However, for $\epsilon>0.005$, and then for high control gains $\boldsymbol{K}$, the control effort $v$ was found to toggle between the two saturation thresholds $\pm 10[N]$. Nevertheless, the tracking of the limit cycle in the presence of the constant uncertainties in the friction parameters was achieved.

\subsection{Third scenario: IWIP under external disturbance}

In this third scenario, we will investigate the effect of the external disturbing torque $\zeta$ on the tracking of the self-generated stable period-1 limit cycle of the IWIP. Moreover, we will assume that the IWIP is without 


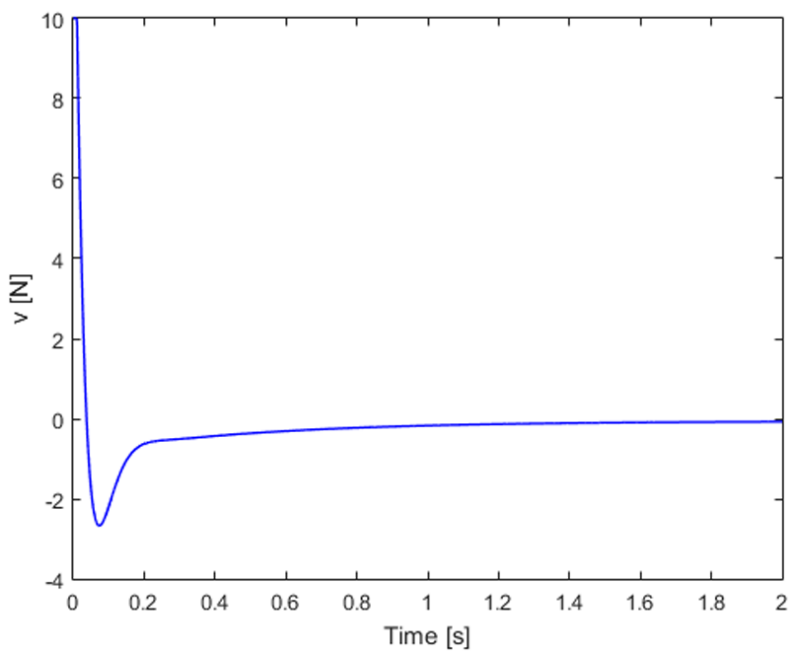

Fig. 7 Tracking control law $v$ for the nominal dynamics of the underactuated IWIP for a first initial condition: $\left(\theta_{1}(0), \theta_{2}(0), \dot{\theta}_{1}(0), \dot{\theta}_{2}(0)\right)=\left(10^{\circ}, 0^{\circ}, 0 \mathrm{rad} / \mathrm{s}, 0 \mathrm{rad} / \mathrm{s}\right)$.

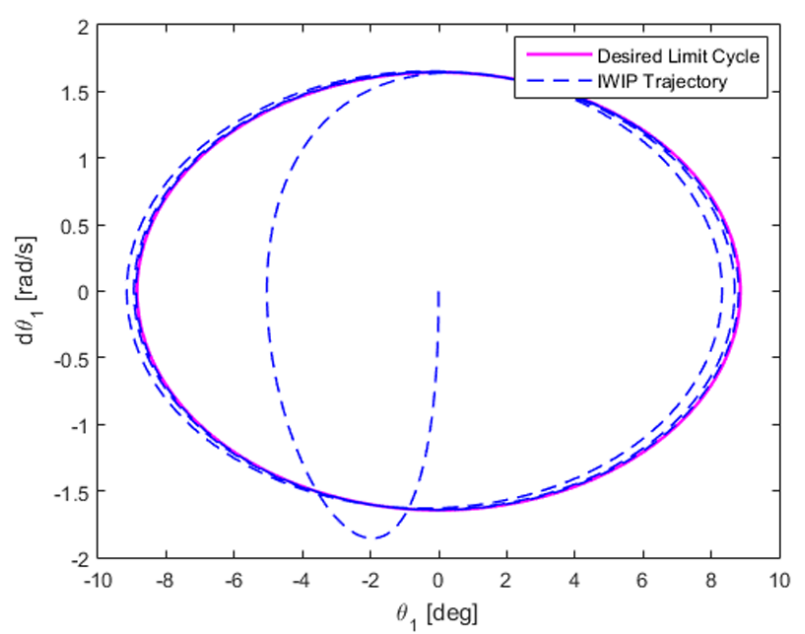

(a)

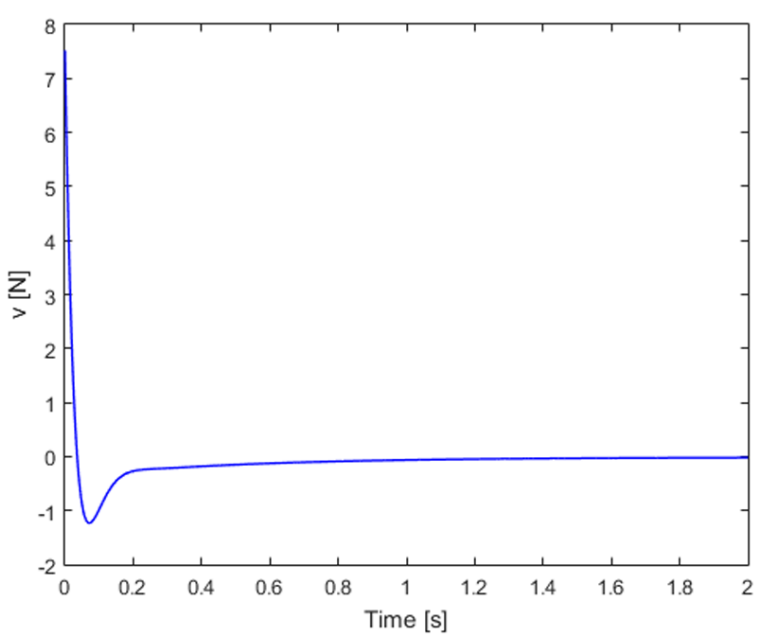

(b)

Fig. 8 Simulation results of the self-generated limit cycle tracking in the nominal case for a new initial condition: $\left(\theta_{1}(0), \theta_{2}(0), \dot{\theta}_{1}(0), \dot{\theta}_{2}(0)\right)=\left(0^{\circ}, 0^{\circ}, 0 \mathrm{rad} / \mathrm{s}, 0 \mathrm{rad} / \mathrm{s}\right)$.

uncertainties, i.e. $\delta_{1}=\delta_{2}=0$. We recall that the dynamics of the controlled IWIP under the disturbance $\zeta$ is given by (17). This disturbance is applied on the pendulum body. Furthermore, we assume that $|\zeta| \leq \rho$, with $\rho>0$. We will consider two cases: a constant disturbing torque $\zeta=\rho$, and a randomly time-varying disturbing torque $\zeta=\rho(2$ Rand -1$)$, with Rand is the function that generates random values between 0 and 1 . In this analysis, the initial condition of the simulation is $\left(\theta_{1}(0), \theta_{2}(0), \dot{\theta}_{1}(0), \dot{\theta}_{2}(0)\right)=\left(10^{\circ}, 0^{\circ}, 0 \mathrm{rad} / \mathrm{s}, 0 \mathrm{rad} / \mathrm{s}\right)$. In addition, the disturbance $\zeta$ is applied during $3[s]$ at the instant $t=2[s]$. Moreover, as in the previous study, the gain matrix $\boldsymbol{K}$ is chosen to be that calculated for the parameter $\epsilon=0.005$ in Table 2 .

\subsubsection{Case 1: Constant disturbance}

In this first scenario, the value of the disturbing torque $\zeta$ applied to the controlled IWIP is constant: $\zeta=\rho$. Figure 11 shows simulation results obtained for two different values of $\rho$. Figs. 11(a)-11(d) reveal the tracking under the disturbance $\zeta=\rho=0.5[N]$, whereas Fig. 11(e)-Fig. 11(h) depict the self-generated stable limit cycle tracking under the constant disturbance torque $\zeta=\rho=1.5[N]$. It is obvious that initially the desired trajectory (stable period-1 limit cycle) of the controlled IWIP was tracked. Once the disturbance $\zeta$ was applied on the IWIP, its behavior will be completely different. The motion of the pendulum and that of the inertia wheel diverge from the desired one. Moreover, it is worth noting that the angular position of the 


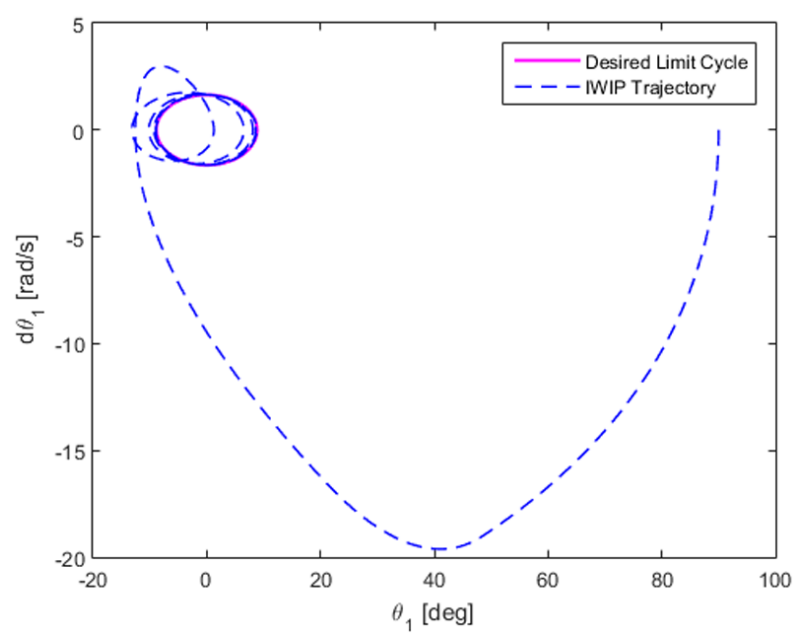

(a)

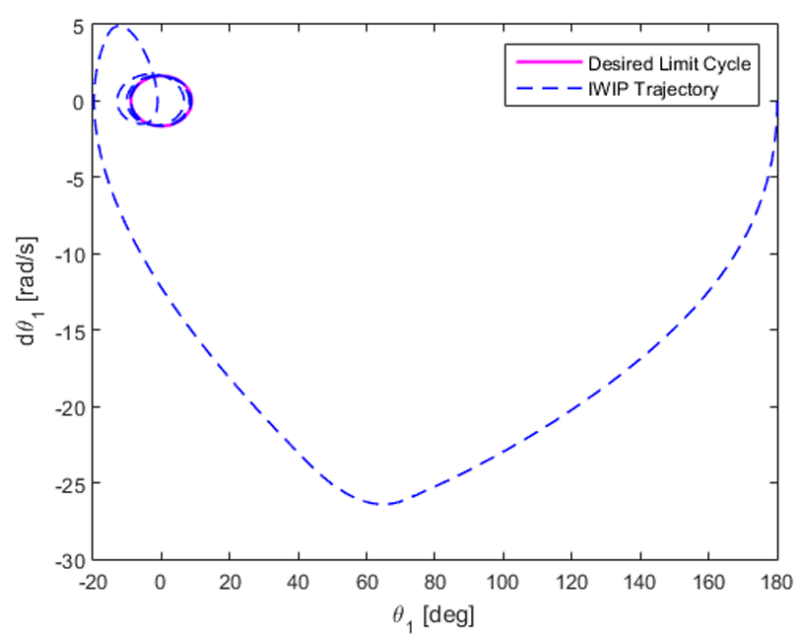

(c)

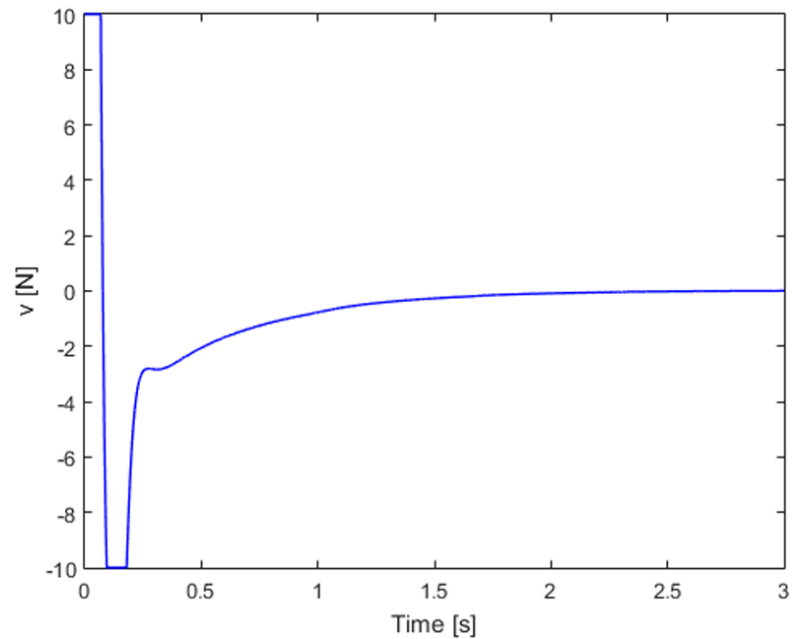

(b)

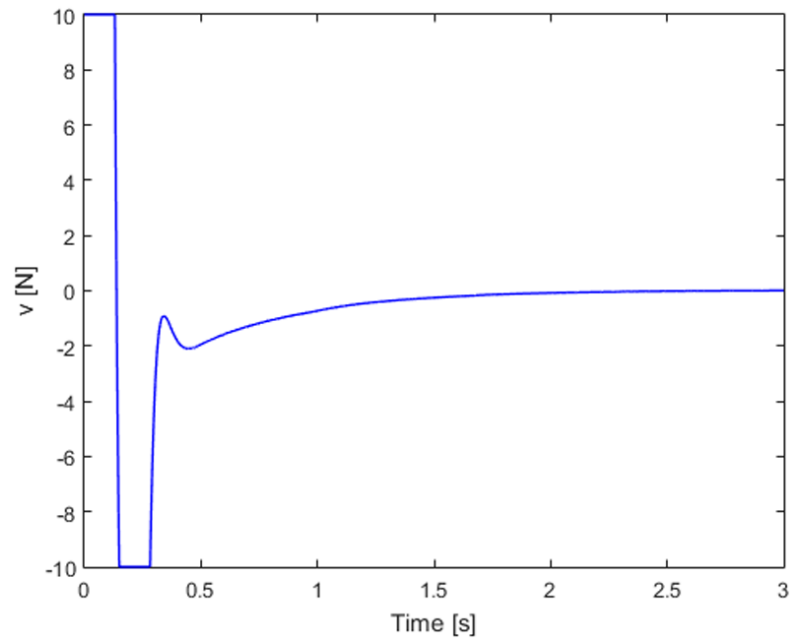

(d)

Fig. 9 Self-generated limit cycle tracking of the underactuated IWIP in the nominal case for two initial conditions $\theta_{1}(0)$ of the inverted pendulum lying outside the defined working state space (1). (a) and (b) for $\theta_{1}(0)=90^{\circ}$. (c) and (d) for $\theta_{1}(0)=180^{\circ}$.

inverted pendulum was found to not respect the unilateral constraints (1) (see Figs. 11(a) and 11(e)). This result, although it is not real for the case of the physical device of the IWIP under study in this work, shows that the proposed controller $v$ was found to be able to bring the trajectory of the IWIP to the desired one despite the presence of the constant disturbance $\zeta$. Moreover, we emphasize that for $\zeta=\rho=0.5[N]$, the control law $v$ converges to a constant value about $-6[N]$ when the IWIP is under disturbance. However, if $\zeta=\rho=1.5[N]$ then the control law was found to be saturated at $-10[N]$. Once the disturbance vanishes, the tracking was achieved again and the controller $v$ becomes almost zero.

We stress that for $\rho>2[N]$ the motion of the controlled IWIP diverges completely from the desired one. The controller was found to be not able to bring the motion of the IWIP to the period- 1 stable limit cycle when the disturbance was canceled.

\subsubsection{Case 2: Randomly time-varying disturbance}

In this second case, the external disturbing torque $\zeta$ varies randomly in time: $\zeta=\rho(2$ Rand -1$)$, with Rand is the function that generates random values between 0 and 1 . Thus, $-\rho \leq \zeta \leq \rho$. Figure 12 reveal simulation results for the controlled IWIP subject to the randomly time-varying external disturbing torque $\zeta$. In this analysis, we have chosen $\rho=5$. It is obvious that when the disturbance vanishes, the motion of the controlled IWIP returns to the period-1 stable oscillation. Moreover, the controller was found to compensate the effect 


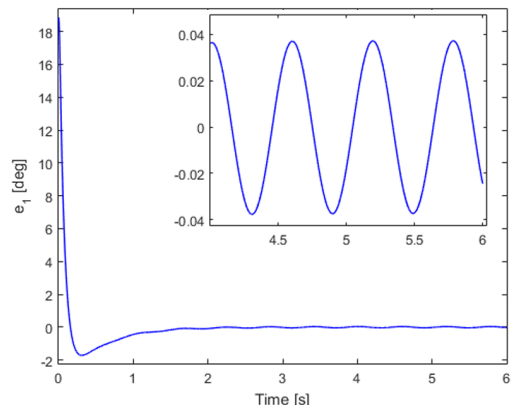

(a)

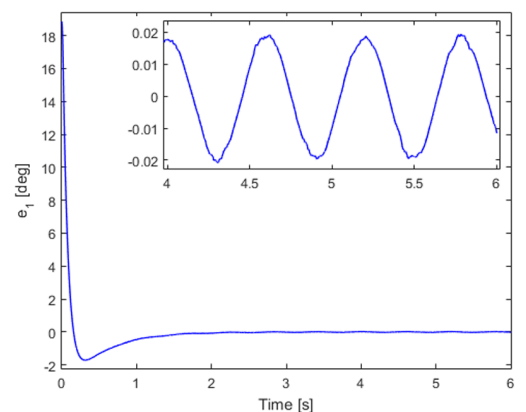

(d)

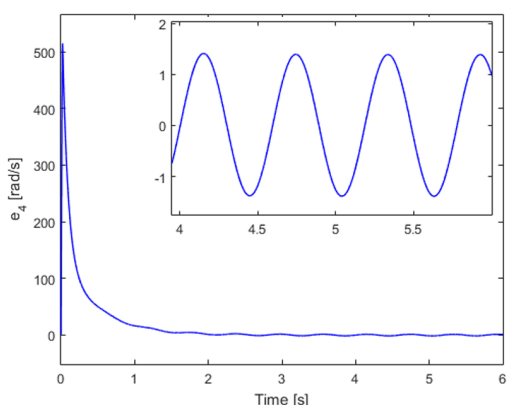

(b)

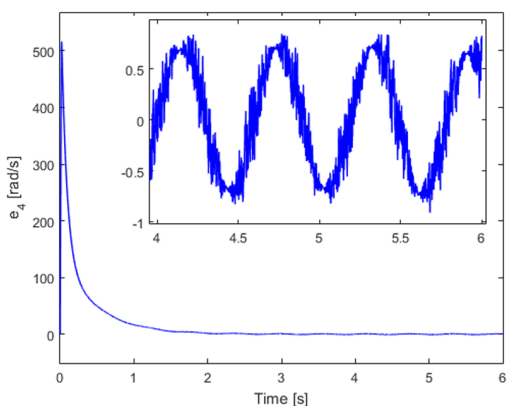

(e)

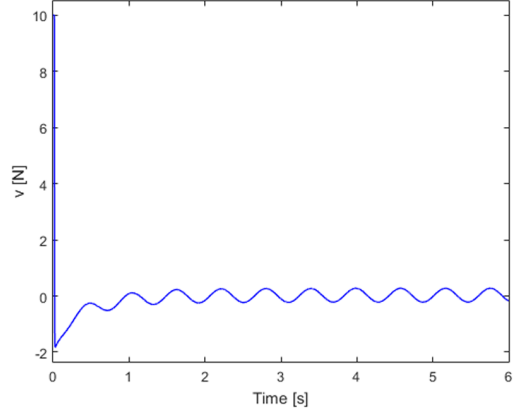

(c)

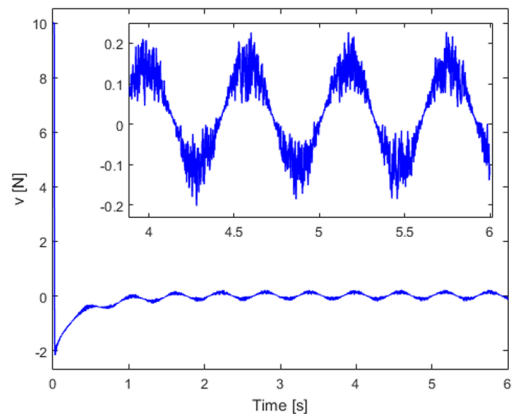

(f)

Fig. 10 Simulation results for the limit cycle tracking of the controlled IWIP for two different cases of frictions $\delta_{1}$ and $\delta_{2}$. In (a)-(c), $\delta_{1}=\delta_{2}=10^{-3}$. Whereas in (d)-(f), $\delta_{1}$ and $\delta_{2}$ are randomly time-varying. (a) and (d) show the tracking error $e_{1}$ of the angular position of the inverted pendulum. (b) and (e) show the tracking error $e_{4}$ of the angular velocity of the inertia wheel. (c) and (f) reveal the tracking control law $v$.

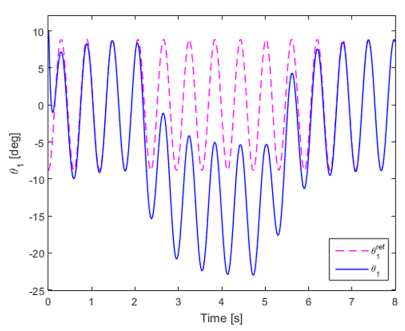

(a)

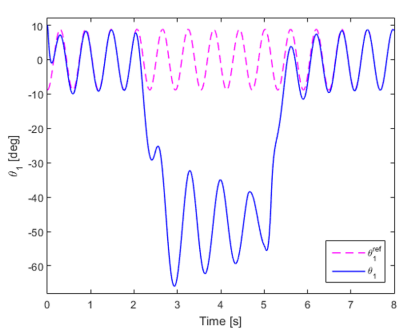

(e)

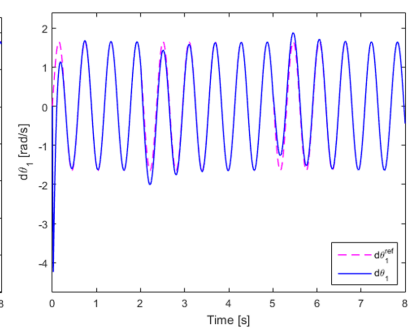

(b)

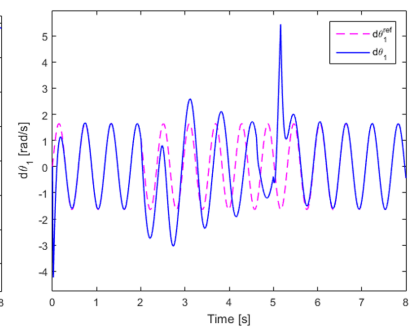

(f)

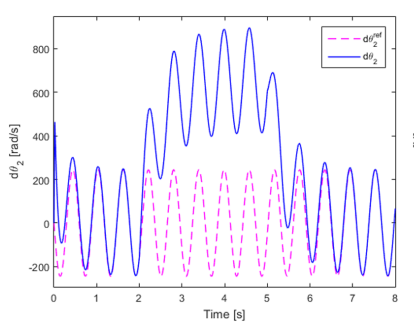

(c)

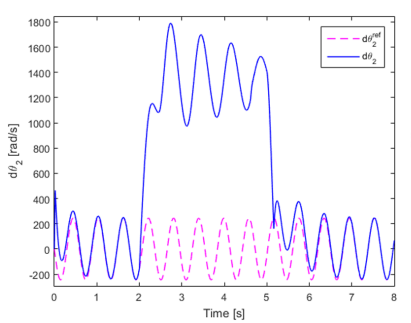

(g)

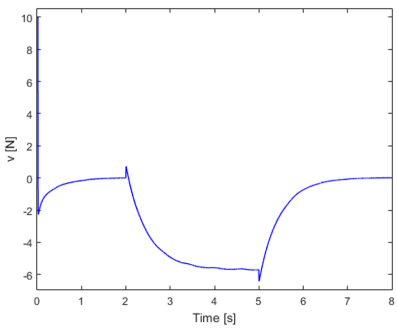

(d)

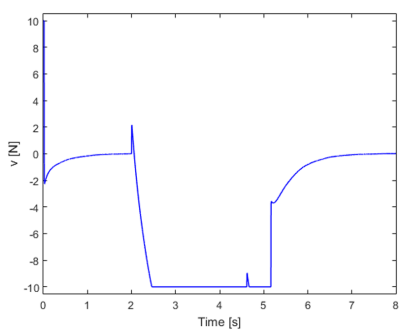

(h)

Fig. 11 Simulation results for the limit cycle tracking of the controlled IWIP under a constant disturbance torque $\zeta$. In (a) $-(\mathrm{d}), \zeta=\rho=0.5[N]$, whereas in (e)-(h), $\zeta=\rho=1.5[N]$. (a) and (e) show the angular position of the inverted pendulum. (b) and (f) depict the angular velocity of the inverted pendulum. (c) and (g) represent the angular velocity of the inertia wheel. (d) and (h) reveal the tracking control law $v$.

of the external disturbing torque $\zeta$. We stress that for $\rho<5$, we have obtained almost the same results except the effect of the disturbance $\zeta$ decreases as $\rho$ decreases.

We note that in Fig. 11 and Fig. 12, the motion area of pendulum (angular position $\theta_{1}$ in Fig. 11(a) and Fig. 12(a)) is larger than $10^{\circ}$. This fact contradicts with the motion constraint (1). In fact, in the numerical simulation when the external disturbance $\zeta$ is applied, the condition (1) was not taken into account because 


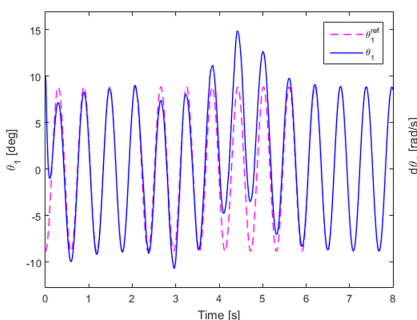

(a)

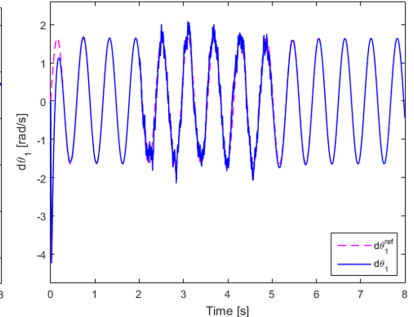

(b)

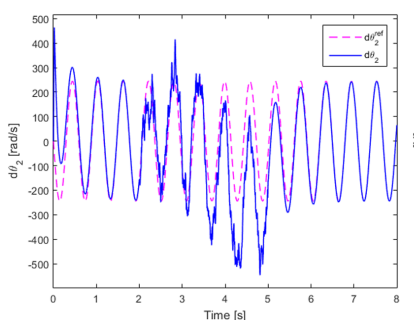

(c)

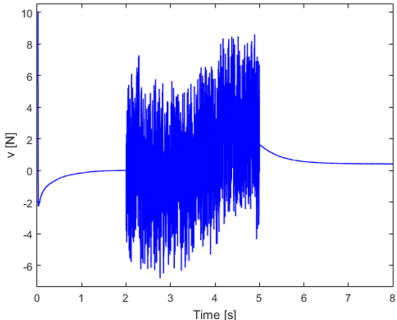

(d)

Fig. 12 Simulation results for the limit cycle tracking of the controlled IWIP subject to a randomly time-varying external disturbing torque $\zeta$. The maximal amplitude of such disturbance is $\rho=5$. (a) shows the angular position of the inverted pendulum. (b) reveals the angular velocity of the inverted pendulum. (c) shows the angular velocity of the inertia wheel. (d) represents the control law $v$.

our main objective is to show the ability of the controlled IWIP to re-track the desired self-generated limit cycle even if it is subject to an external disturbance with a large amplitude under which the IWIP is very far from the desired one (as in Fig. 10 with an initial condition outside the required region). Thus, if we take the state constraint (1) into consideration, then under the external disturbance, the pendulum will stuck (with some small oscillations) to the left/right mechanical stop until the disturbance is canceled or another external disturbance with small amplitude is applied. Hence, we will have $\theta_{1}=10^{\circ}$ for some stretches of time.

Remark 6 It is worth pointing out that the choice of the external disturbing torque $\zeta$ to be randomly timevarying is arbitrary. It can be seen as a vibration signal applied to the IWIP. We can choose a regular form of the disturbing torque $\zeta$, for example $\zeta(t)=A_{\zeta} \sin \left(w_{\zeta} t\right)$, where $A_{\zeta}$ and $w_{\zeta}$ are respectively the amplitude and the frequency of the disturbing torque $\zeta$. Thus, we obtained almost the same results. When the disturbance was canceled, the controlled IWIP returns to its self-generated stable limit cycle. Moreover, we can choose another regular form of the disturbing torque such as: $\zeta(t)=A_{\zeta} e\left(-\lambda_{\zeta} t\right)$. Here, the disturbing torque $\zeta$ will vanish progressively in time. We obtained also the same behavior of the controlled IWIP.

\section{Conclusion and future works}

In this paper, a control design approach is proposed for the tracking of a stable period-1 limit cycle of an underactuated mechanical system: the inertia wheel inverted pendulum (IWIP). Such system is subject to unilateral constraints limiting the swing motion of the pendulum body around its unstable equilibrium point. Moreover, an IDA-PBC was applied to the IWIP to control the underactuated IWIP at its upright position. By analyzing stability of the equilibrium point of the IWIP under the IDA-PBC and then existence of the Hopf bifurcation, we showed that the controlled IWIP generated a stable limit cycle.

For some value of the gain parameter of the IDA-PBC, the nonlinear dynamics of the controlled IWIP was adopted as the reference model generating the period-1 stable limit cycle, which is used as the tracking trajectory for the IWIP. Thus, the tracking problem was transformed into a stabilization problem of the tracking error. We used first a predefined control law, which gave us a simplified model. Moreover, we adopted a state-feedback linear control law to asymptotically stabilize the tracking error. For this purpose, our methodology was based on the use of the second Lyapunov method and the S-procedure lemma. Thus, the stabilization problem was converted into a solving problem of BMIs. We employed the Schur complement lemma and the matrix inversion lemma in order to transform such BMIs into LMIs. Finally, several simulations have been presented to corroborate the mathematical results and hence to show the feasibility of the designed controller for the tracking of the self-generated limit cycle of the IWIP under the IDA-PBC. We analyzed three different scenarios: (1) a nominal dynamics of the IWIP, i.e. without uncertainties in parameters and without disturbance, (2) IWIP under constant and randomly time-varying uncertain friction parameters, and (3) IWIP subject to constant and randomly time-varying external disturbing torque applied on the pendulum body. Simulation results showed that the control law was able, for some defined control gain and a reasonable amplitude of the external disturbance, to compensate the effects of the uncertainties in the friction parameters and the external disturbance.

In this work, the control scheme is designed in the special case of the IWIP. However, our methodology for limit cycle tracking can be easily generalized to the more general case of underactuated mechanical flat systems [12]. Moreover, as a future direction, we look for designing a robust tracking controller for the 
IWIP with uncertain parameters and/or under external disturbances using the nonlinear dynamics (17). In addition, we look for designing an observer or an observer-based controller to control the IWIP to its upright position or to track a desired trajectory.

Furthermore, we aim at verifying the obtained results in simulation via real-time experiments using the constrained IWIP as in [34, 35, 53, 54] or that employed by [33].

Moreover, we showed that the control effort on the IWIP displayed oscillations if a constant friction parameter is introduced. Thus, using the theory of the Hopf bifurcation as realized in this paper for the generation of periodic oscillations by varying the parameters of the IDA-PBC controller, we can demonstrate theoretically the birth of these oscillations by varying the friction parameter. This will be considered as our main future work.

In addition, we aim at extending our developed method of the state-feedback controller for the robust tracking of a desired limit cycle to more complex nonlinear systems. Our concern will be impulsive hybrid nonlinear dynamics of biped robots studied by us in [88-90], and more particularly the passive dynamic walking of the planar compass-gait biped robot [88] and the semi-passive dynamic walking of the torsodriven biped robot $[89,90]$. Our design methodology of the tracking controller will be based on the LMI techniques.

\section{Appendix 1: Self-generation of a limit cycle through Hopf bifurcation}

In this Appendix, we show generation of a self-sustained oscillation (limit cycle) in the nonlinear dynamics of the underactuated IWIP under the IDA-PBC. Thus, we present first stability conditions of the equilibrium point $\boldsymbol{x}_{e q}$, given by expression (9), of the nonlinear dynamics (8). Moreover, we derive conditions proving existence of the Hopf bifurcation and hence the periodic solutions in the nonlinear dynamics (8). Finally, we discuss briefly stability of the periodic solutions using the fist Lyapunov value [4]. This quantity is calculated numerically in this paper. Its mathematical description will be developed and presented in another new paper. It is worth to note that analysis of $3 \mathrm{D}$ or $4 \mathrm{D}$ nonlinear dynamics has been widely investigated, as in [91-96], just to mention a few.

Appendix 1.1: Stability of the equilibrium point

We look for determining conditions on the control gains $k_{i}(i=1,2,3,4)$ for which the equilibrium point is stable and hence the Hopf bifurcation occurs.

For simplicity, posing $a=\frac{1}{I}, b=b g$ and $c=\frac{1}{I_{2}}$. Then, the characteristic equation of the Jacobian matrix (10) is defined as:

$$
\mathcal{P}(\lambda)=\lambda^{4}+\left(k_{3}-k_{4}\right) \lambda^{3}+\left(a\left(\gamma-b+k_{1}\right)-c k_{2}\right) \lambda^{2}+a b k_{4} \lambda+a b c k_{2}=0
$$

In order to study the stability conditions of the equilibrium point $\boldsymbol{x}_{e q}$, we apply Routh-Hurwitz criterion, which states that all real eigenvalues and all real parts of complex conjugate eigenvalues of the characteristic polynomial $\mathcal{P}(\lambda)$ are negative if and only if the following conditions hold:

$$
\text { (i) } a_{1}>0 \text {, (ii) } a_{3}>0 \text {, (iii) } a_{4}>0 \text { and (iv) } a_{1} a_{2} a_{3}>a_{3}^{2}+a_{1}^{2} a_{4}
$$

with $a_{i}(i=1,2,3,4)$, are defined such that:

$$
\mathcal{P}(\lambda)=\lambda^{4}+a_{1} \lambda^{3}+a_{2} \lambda^{2}+a_{3} \lambda+a_{4}=0
$$

Applying Routh-Hurwitz criterion (75) to the characteristic polynomial (74), and as $k_{i}>0$ for $i=$ $1,2,3,4$, we find that necessary and sufficient conditions for the equilibrium point $\boldsymbol{x}_{e q}$ to be asymptotically stable are:

$$
\begin{gathered}
\mathcal{C}_{1}=k_{3}-k_{4}>0 \\
\mathcal{C}_{2}=a\left(\gamma-b+k_{1}\right)-c k_{2}-a b \frac{k_{4}}{k_{3}-k_{4}}-c k_{2} \frac{k_{3}-k_{4}}{k_{4}}>0
\end{gathered}
$$


Appendix 1.2: Existence of Hopf bifurcation and periodic solutions

In this part, we will investigate existence of the Hopf bifurcation [4] at the equilibrium point regarding $k_{4}$ as the bifurcation parameter. Assume that (74) has a pure imaginary root $\lambda=\mathrm{i} w, w \in \mathbb{R}^{+}$. Substituting it into (74) yields

$$
w^{4}-\left(a\left(\gamma-b+k_{1}\right)-c k_{2}\right) w^{2}+a b c k_{2}+\mathrm{i} w\left(-\left(k_{3}-k_{4}\right) w^{2}+a b k_{4}\right)=0
$$

It then follows that:

$$
\begin{gathered}
w^{4}-\left(a\left(\gamma-b+k_{1}\right)-c k_{2}\right) w^{2}+a b c k_{2}=0 \\
\left(k_{4}-k_{3}\right) w^{2}+a b k_{4}=0
\end{gathered}
$$

From relation $(79 \mathrm{~b})$, we obtain the following expression:

$$
k_{4}=\frac{w^{2}}{a b+w^{2}} k_{3}
$$

We stress that $w$ is the solution of (79a) and it depends only on the two parameters $k_{1}$ and $k_{2}$. Moreover, according to expression (80), $k_{4}$ is proportional to $k_{3}$ and it depends on $w$. Then, by fixing the value of the parameters $k_{1}, k_{2}$ and $k_{3}$, we can calculate the value of the gain $k_{4}$ at which the Hopf bifurcation occurs.

By solving relation (79a), it is easy to show that there are two solutions $w_{1}^{c}$ and $w_{2}^{c}$ defined as follows:

$$
\begin{aligned}
& w_{1}^{c}=\sqrt{\frac{a\left(\gamma-b+k_{1}\right)-c k_{2}-\sqrt{\left(a\left(\gamma-b+k_{1}\right)-c k_{2}\right)^{2}-4 a b c k_{2}}}{2}} \\
& w_{2}^{c}=\sqrt{\frac{a\left(\gamma-b+k_{1}\right)-c k_{2}+\sqrt{\left(a\left(\gamma-b+k_{1}\right)-c k_{2}\right)^{2}-4 a b c k_{2}}}{2}}
\end{aligned}
$$

In (81), we have $w_{1}^{c}<w_{2}^{c}$. Actually, these two solutions, $w_{1}^{c}$ and $w_{2}^{c}$, exist if and only if the following three conditions hold:

$$
\begin{gathered}
\left(a\left(\gamma-b+k_{1}\right)-c k_{2}\right)^{2}-4 a b c k_{2}>0 \\
a\left(\gamma-b+k_{1}\right)-c k_{2}-\sqrt{\left(a\left(\gamma-b+k_{1}\right)-c k_{2}\right)^{2}-4 a b c k_{2}}>0 \\
a\left(\gamma-b+k_{1}\right)-c k_{2}+\sqrt{\left(a\left(\gamma-b+k_{1}\right)-c k_{2}\right)^{2}-4 a b c k_{2}}>0
\end{gathered}
$$

As $k_{1}>0$ and $k_{2}>0$, then according to conditions (82b) and (82c), it follows that: $a\left(\gamma-b+k_{1}\right)-c k_{2}>0$. Therefore, relying on (82a), it easy to show that:

$$
k_{1}>b-\gamma+\frac{c}{a} k_{2}+2 \sqrt{\frac{b c}{a}} \sqrt{k_{2}}
$$

This expression represents the condition on $k_{1}$ with respect to $k_{2}$ for the existence of the Hopf bifurcation. In addition, we emphasize that the system (8) exhibits two Hopf bifurcations at two different critical values $k_{4,1}^{c}$ and $k_{4,2}^{c}$ that are associated to $w_{1}^{c}$ and $w_{2}^{c}$, respectively. These two critical parameters $k_{4,1}^{c}$ and $k_{4,2}^{c}$ are defined like so:

$$
\begin{aligned}
& k_{4,1}^{c}=k_{3} \frac{a\left(\gamma-b+k_{1}\right)-c k_{2}-\sqrt{\left(a\left(\gamma-b+k_{1}\right)-c k_{2}\right)^{2}-4 a b c k_{2}}}{a\left(\gamma+b+k_{1}\right)-c k_{2}-\sqrt{\left(a\left(\gamma-b+k_{1}\right)-c k_{2}\right)^{2}-4 a b c k_{2}}} \\
& k_{4,2}^{c}=k_{3} \frac{a\left(\gamma-b+k_{1}\right)-c k_{2}+\sqrt{\left(a\left(\gamma-b+k_{1}\right)-c k_{2}\right)^{2}-4 a b c k_{2}}}{a\left(\gamma+b+k_{1}\right)-c k_{2}+\sqrt{\left(a\left(\gamma-b+k_{1}\right)-c k_{2}\right)^{2}-4 a b c k_{2}}}
\end{aligned}
$$

For example, for $k_{1}=1.036692, k_{2}=0.0011$ and $k_{3}=38.4$, the two critical values of $k_{4}$ at which the Hopf bifurcation occurs are calculated to be about: $k_{4,1}^{c}=0.87159917$ and $k_{4,2}^{c}=27.19772529$. Moreover, we have: $w_{1}^{c}=1.04160466$ and $w_{2}^{c}=10.64970853$. 
It is worth noting that for $k_{4}=k_{4, i}^{c},(i=1,2)$, the relation $\mathcal{C}_{2}=0$ in $(77 \mathrm{~b})$ takes place, for which the stability of the equilibrium is violated. Moreover, if the condition (83) is not satisfied, the two Hopf bifurcations disappear. In fact, there are two cases. The two Hopf bifurcations collide with each other and are replaced by only one Hopf bifurcation if the following condition is met:

$$
k_{1}=b-\gamma+\frac{c}{a} k_{2}+2 \sqrt{\frac{b c}{a}} \sqrt{k_{2}}
$$

Hence, for such expression (90), we will obtain: $w_{1}^{c}=w_{2}^{c}=w_{\star}^{c}=\sqrt{\sqrt{a b c} \sqrt{k_{2}}}$, and then:

$$
k_{4, \star}^{c}=k_{3} \frac{\sqrt{c k_{2}}}{\sqrt{a b}+\sqrt{c k_{2}}}
$$

Moreover, if $k_{1}<b-\gamma+\frac{c}{a} k_{2}+2 \sqrt{\frac{b c}{a}} \sqrt{k_{2}}$, then no Hopf bifurcation exits.

Now, we aim at developing the analytical expression of the transversality condition for the occurrence of the Hopf bifurcation. From (74), it follows that:

$$
\frac{d \lambda\left(k_{4}\right)}{d k_{4}}=\frac{\mathrm{i} w\left(w^{2}+a b\right)}{a b k_{4}-3\left(k_{3}-k_{4}\right) w^{2}+2 \mathrm{i} w\left(a\left(\gamma-b+k_{1}\right)-c k_{2}-2 w^{2}\right)}
$$

Thus, we obtain:

$$
\operatorname{Re}\left(\frac{d \lambda\left(k_{4}\right)}{d k_{4}}\right)=\frac{2 w^{2}\left(w^{2}+a b\right)\left(a\left(\gamma-b+k_{1}\right)-c k_{2}-2 w^{2}\right)}{\left(a b k_{4}-3\left(k_{3}-k_{4}\right) w^{2}\right)^{2}+4 w^{2}\left(a\left(\gamma-b+k_{1}\right)-c k_{2}-2 w^{2}\right)^{2}}
$$

It is clear that the sign of the condition (88) depends on the quantity $\vartheta=a\left(\gamma-b+k_{1}\right)-c k_{2}-2 w^{2}$. Using expressions of $w_{1}^{c}$ and $w_{2}^{c}$, it follows that: $\vartheta=\sqrt{\left(a\left(\gamma-b+k_{1}\right)-c k_{2}\right)^{2}-4 a b c k_{2}}$ for $w=w_{1}^{c}$, and $\vartheta=-\sqrt{\left(a\left(\gamma-b+k_{1}\right)-c k_{2}\right)^{2}-4 a b c k_{2}}$ for $w=w_{2}^{c}$. Furthermore, based on condition (83), we obtain $\vartheta \neq 0$ for the two cases. Therefore,

$$
\operatorname{Re}\left(\left.\frac{d \lambda\left(k_{4}\right)}{d k_{4}}\right|_{k_{4}=k_{4, i}^{c}, w=w_{i}^{c}}\right) \neq 0
$$

Accordingly, the second condition for the existence of a Hopf bifurcation [4] is also met. In addition, we have:

$$
\begin{aligned}
& \operatorname{Re}\left(\left.\frac{d \lambda\left(k_{4}\right)}{d k_{4}}\right|_{k_{4}=k_{4,1}^{c}, w=w_{1}^{c}}\right)>0 \\
& \operatorname{Re}\left(\left.\frac{d \lambda\left(k_{4}\right)}{d k_{4}}\right|_{k_{4}=k_{4,2}^{c}, w=w_{2}^{c}}\right)<0
\end{aligned}
$$

Hence, according to condition (90a) the equilibrium point is stable when $k_{4}>k_{4,1}^{c}$ and periodic solutions exist when $k_{4}<k_{4,1}^{c}$. Furthermore, according to condition (90b) the equilibrium point is stable when $k_{4}<k_{4,2}^{c}$ and periodic solutions exist when $k_{4}>k_{4,2}^{c}$. Furthermore, the period $T_{i}$ of oscillations around the bifurcation point $k_{4, i}^{c}$ is defined as follows:

$$
T_{i}=\frac{2 \pi}{w_{i}^{c}}
$$

Appendix 1.3: Stability of the periodic solutions

In order to characterize the stability of the periodic solutions generated through each Hopf bifurcation, the so-called fist Lyapunov value (coefficient) [4], say $\ell_{1}$, must be computed and analyzed for the system (8). In the bifurcation points, a positive first Lyapunov value represents a subcritical bifurcation and predicts that the system presents unstable periodic oscillations, which can fold back and coexist with the stable equilibrium point. In contrast, a negative value for $\ell_{1}$ indicates a supercritical bifurcation and stable selfoscillations emerge from the bifurcation point.

Analytical calculation of the fist Lyapunov coefficient $\ell_{1}$ can be achieved, but it is very long. It can be calculated according to the formula given in [97]. For the sake of brevity, we omit this calculation in this paper. In fact, this analysis on stability of the periodic oscillations and bifurcation through the first Lyapunov 
value and the center manifold theory will be developed with further details in another work. Then, in this study we will perform a numerical computation of $\ell_{1}$ using the well-known MATCONT package [97, 98]. Alonso et al. $[57,58]$ used the AUTO package to the numerical continuation of limit cycles and the stability index for the underactuated IWP.

Thus, using MATCONT package, the fist Lyapunov coefficient $\ell_{1}$ is computed to be: $\ell_{1,1}=3.121836 \times$ $10^{-9}$ at $k_{4,1}^{c}=0.87159917$, whereas $\ell_{1,2}=-1.081602 \times 10^{-5}$ at $k_{4,2}^{c}=27.19772529$. Therefore, as $\ell_{1,1}>0$ then the periodic solution emanating from the first Hopf bifurcation at $k_{4,1}^{c}$ is unstable, and which arises at the side where the equilibrium is stable. However, because $\ell_{1,2}<0$ hence the generated sustained oscillation at the parameter $k_{4,2}^{c}$ is stable. Moreover, this stable periodic oscillation arises at the side where the equilibrium is unstable. Relying on relation (91), the period of the stable self-sustained oscillation (stable limit cycle) around the critical parameter $k_{4,2}^{c}$ is: $T_{2}=0.5899866[s]$.

\section{Appendix 2: Equivalent BMI (29b) for a IWIP with uncertain friction parameters and an external} disturbance

Using the reference dynamics (16) and the uncertain disturbed nonlinear dynamics (17), we obtain the following tracking error dynamics:

$$
\dot{\boldsymbol{e}}=\boldsymbol{J} \boldsymbol{e}+\boldsymbol{D}\left(f\left(x_{1}\right)-f\left(x_{1}^{r}\right)\right)+\boldsymbol{B} v+\tilde{\boldsymbol{J}} \boldsymbol{x}+\tilde{\boldsymbol{D}} \zeta
$$

By substituting the tracking control law (20) in (92), system (92) will be simplified as follows:

$$
\dot{\overline{\boldsymbol{e}}}=\boldsymbol{A} \overline{\boldsymbol{e}}+\overline{\boldsymbol{D}}\left(f\left(x_{1}\right)-f\left(x_{1}^{r}\right)\right)+\overline{\boldsymbol{B}} w+\tilde{\boldsymbol{A}} \boldsymbol{x}+\boldsymbol{D}_{5} \zeta
$$

with $\tilde{\boldsymbol{A}}=\left[\begin{array}{ccc}0 & 0 & 0 \\ 0 & -\frac{\delta_{1}+\delta_{2}}{I} & \frac{\delta_{2}}{I_{2}} \\ 0 & \frac{\delta_{2}}{I} & -\frac{\delta_{2}}{I_{2}}\end{array}\right]$ and $\boldsymbol{D}_{5}=\left[\begin{array}{l}0 \\ 1 \\ 0\end{array}\right]$

Moreover, the term $\tilde{\boldsymbol{A}} \boldsymbol{x}$ in (93) can be rewritten like so: $\tilde{\boldsymbol{A}} \boldsymbol{x}=\delta_{1} \boldsymbol{D}_{2} x_{3}+\delta_{2} \boldsymbol{D}_{3} x_{3}+\delta_{2} \boldsymbol{D}_{4} x_{4}$, with $\boldsymbol{D}_{2}=\frac{1}{I}\left[\begin{array}{c}0 \\ -1 \\ 0\end{array}\right], \boldsymbol{D}_{3}=\frac{1}{I}\left[\begin{array}{c}0 \\ -1 \\ 1\end{array}\right], \boldsymbol{D}_{4}=\frac{1}{I_{2}}\left[\begin{array}{c}0 \\ 1 \\ -1\end{array}\right]$.

In addition, if we consider that:

- the two uncertain friction parameters $\delta_{1}$ and $\delta_{2}$ are norm-bounded, i.e. $\delta_{1} \leq \bar{\delta}_{1}$ and $\delta_{2} \leq \bar{\delta}_{2}$,

- the two states $x_{3}$ and $x_{4}$ are bounded such that $\left|x_{3}\right|<\bar{x}_{3}$ and $\left|x_{4}\right|<\bar{x}_{4}$, for some constants $\bar{x}_{3}>0$ and $\bar{x}_{4}>0$,

- the disturbance $\zeta$ is bounded such that $|\zeta|<\rho$, with $\rho>0$.

then, according to section 4.2 and relying on the Young relation, we obtain the following conditions:

$$
\begin{gathered}
2 \overline{\boldsymbol{e}}^{\mathrm{T}} \boldsymbol{P} \tilde{\boldsymbol{A}} \boldsymbol{x} \leq \overline{\boldsymbol{e}}^{\mathrm{T}} \boldsymbol{P}\left(\boldsymbol{M}_{2}+\boldsymbol{M}_{3}+\boldsymbol{M}_{4}\right) \boldsymbol{P} \overline{\boldsymbol{e}}+\left(\bar{\delta}_{1} \bar{x}_{3}\right)^{2} \boldsymbol{D}_{2}^{\mathrm{T}} \boldsymbol{M}_{2}^{-1} \boldsymbol{D}_{2}+\left(\bar{\delta}_{2} \bar{x}_{3}\right)^{2} \boldsymbol{D}_{3}^{\mathrm{T}} \boldsymbol{M}_{3}^{-1} \boldsymbol{D}_{3}+\left(\bar{\delta}_{2} \bar{x}_{4}\right)^{2} \boldsymbol{D}_{4}^{\mathrm{T}} \boldsymbol{M}_{4}^{-1} \boldsymbol{D}_{4} \\
2 \overline{\boldsymbol{e}}^{\mathrm{T}} \boldsymbol{P} \boldsymbol{D}_{5} \zeta \leq \overline{\boldsymbol{e}}^{\mathrm{T}} \boldsymbol{P} \boldsymbol{M}_{5} \boldsymbol{P} \overline{\boldsymbol{e}}+\rho^{2} \boldsymbol{D}_{5}^{\mathrm{T}} \boldsymbol{M}_{5}^{-1} \boldsymbol{D}_{5}
\end{gathered}
$$

with $\boldsymbol{M}_{i}=\boldsymbol{M}_{i}^{\mathrm{T}}>0, i=2, \ldots, 5$.

$$
\text { Let us pose } \boldsymbol{J}=\left[\begin{array}{c}
\mathcal{I} \\
\mathcal{I} \\
\mathcal{I} \\
\mathcal{I} \\
\mathcal{I}
\end{array}\right], \boldsymbol{M}=\operatorname{diag}\left(\boldsymbol{M}_{1}, \boldsymbol{M}_{2}, \boldsymbol{M}_{3}, \boldsymbol{M}_{4}, \boldsymbol{M}_{5}\right) \text {, and } \boldsymbol{D}=\left[\begin{array}{c}
\gamma \overline{\boldsymbol{D}} \\
\bar{\delta}_{1} \bar{x}_{3} \boldsymbol{D}_{2} \\
\bar{\delta}_{2} \bar{x}_{3} \boldsymbol{D}_{3} \\
\bar{\delta}_{2} \bar{x}_{4} \boldsymbol{D}_{4} \\
\boldsymbol{D}_{5}
\end{array}\right] \text {. Thus, based on results }
$$

in section 4.2 , the BMI (29b) will be replaced by the following one:

$$
\left[\begin{array}{cc}
\boldsymbol{P} \boldsymbol{A}+\boldsymbol{P} \overline{\boldsymbol{B}} \boldsymbol{K}+(\star)+\boldsymbol{P} \boldsymbol{J}^{\mathrm{T}} \boldsymbol{M} \boldsymbol{J} \boldsymbol{P}+\eta_{3} \boldsymbol{I} & -\left(\eta_{1}-\eta_{2}\right) \boldsymbol{C} \\
(\star) & \boldsymbol{D}^{\mathrm{T}} \boldsymbol{M}^{-1} \boldsymbol{D}-\epsilon \eta_{3}+4 \sigma\left(\eta_{1}+\eta_{2}\right)
\end{array}\right]<0
$$




\section{References}

1. M. di Bernardo, K. H. Johansson, F. Vasca, Self-oscillations and sliding in relay feedback systems: Symmetry and bifurcations, International Journal of Bifurcation and Chaos 11 (04) (2001) 1121-1140.

2. A. Jenkins, Self-oscillation, Physics Reports 525 (2) (2013) 167-222.

3. S. Chatterjee, Self-excited oscillation under nonlinear feedback with time-delay, Journal of Sound and Vibration 330 (9) (2011) 1860-1876.

4. J. Guckenheimer, P. Holmes, Nonlinear Oscillations, Dynamical Systems, and Bifurcations of Vector Fields, 1st Edition, Applied Mathematical Sciences, Springer, New York, 1983.

5. J. Sun, A. C. Luo, Bifurcation and Chaos in Complex Nonlinear Dynamical Systems, Advances in Nonlinear Science and Complexity (Physics), Elsevier, 2006.

6. S. H. Strogatz, Nonlinear Dynamics and Chaos: With Applications to Physics, Biology, Chemistry, and Engineering, 2nd Edition, Studies in Nonlinearity, Westview Press, Colorado, 2014.

7. K. T. Alligood, T. D. Sauer, J. A. Yorke, Chaos: An Introduction to Dynamical Systems, 1st Edition, Textbooks in Mathematical Sciences, Springer, New York, 1996.

8. A. Malas, S. Chatterjee, Generating self-excited oscillation in a class of mechanical systems by relayfeedback, Nonlinear Dynamics 76 (2) (2014) 1253-1269.

9. A. Malas, S. Chatterjee, Analysis and synthesis of modal and non-modal self-excited oscillations in a class of mechanical systems with nonlinear velocity feedback, Journal of Sound and Vibration 334 (2015) 296-318.

10. S. Wiggins, Introduction to Applied Nonlinear Dynamical Systems and Chaos, 2nd Edition, Vol. 2 of Texts in Applied Mathematics, Springer, New York, 2003.

11. M. W. Spong, Underactuated mechanical systems, in: B. Siciliano, K. P. Valavanis (Eds.), Control Problems in Robotics and Automation, Vol. 230 of Lecture Notes in Control and Information Sciences, Springer Berlin Heidelberg, 1998, pp. 135-150.

12. Y. Liu, H. Yu, A survey of underactuated mechanical systems, IET Control Theory Applications 7 (7) (2013) 921-935.

13. A. Choukchou-Braham, B. Cherki, M. Djemai, K. Busawon, Analysis and Control of Underactuated Mechanical Systems, Springer-Verlag, New York, 2014.

14. A.-C. Huang, Y.-F. Chen, C.-Y. Kai, Adaptive Control of Underactuated Mechanical Systems, World Scientific Publishing Co, Singapore, 2015.

15. I. Fantoni, R. Lozano, Nonlinear Control for Underactuated Mechanical Systems, Springer, London, 2002.

16. S. Rudra, R. K. Barai, M. Maitra, Block Backstepping Design of Nonlinear State Feedback Control Law for Underactuated Mechanical Systems, 1st Edition, Springer, Singapore, 2017.

17. J. Moreno-Valenzuela, C. Aguilar-Avelar, S. Puga-Guzmán, V. Santibánez, Two adaptive control strategies for trajectory tracking of the inertia wheel pendulum: neural networks vis à vis model regressor, Intelligent Automation \& Soft Computing (2016) 1-11doi:10.1080/10798587.2015.1121618.

18. Z. Li, Y. Zhang, Robust adaptive motion/force control for wheeled inverted pendulums, Automatica 46 (8) (2010) 1346-1353.

19. M. W. Spong, P. Corke, R. Lozano, Nonlinear control of the Reaction Wheel Pendulum, Automatica 37 (2001) 1845-1851.

20. D. J. Block, K. J. Astrom, M. W. Spong, The Reaction Wheel Pendulum, 1st Edition, Synthesis Lectures on Control and Mechatronics, Morgan \& Claypool, San Rafael, 2007.

21. R. Olfati-Saber, Nonlinear control of underactuated mechanical systems with application to robotics and aerospace vehicles, Ph.D. thesis, Massachusetts Institute of Technology, Department Electrical Engineering and Computer Science (2001).

22. R. Olfati-Saber, Global stabilization of a flat underactuated system : the inertia wheel pendulum, in: Proceedings of the CDC01-REG1152, 2001.

23. S. Ramamoorthy, B. Kuipers, Qualitative heterogeneous control of higher order systems, Hybrid Systems: Computation and Control (2003) 417-434.

24. V. Santibanez, R. Kelly, J. Sandoval, Control of the inertia wheel pendulum by bounded torques, in: Proceedings of the European Control Conference and 44th IEEE Conference on Decision and Control, 2005, pp. 8266-8270.

25. R. Ortega, M. Spong, F. Gomez-Estern, G. Blankenstein, Stabilization of a class of underactuated mechanical systems via interconnection and damping assignment, IEEE Transactions on Automatic Control 47 (8) (2002) 1218-1233. 
26. R. Ortega, E. Garcia-Canseco, Interconnection and damping assignment passivity-based control: A survey, European Journal on control 10 (2004) 432-450.

27. R. Ortega, A. van der Schaft, B. Maschke, G. Escobar, Interconnection and damping assignment passivity-based control of port-controlled hamiltonian systems, Automatica 38 (4) (2002) 585-596.

28. F. Gómez-Estern, A. J. Van der Schaft, Physical damping in IDA-PBC controlled underactuated systems, European Journal on control 10 (5) (2004) 451-468.

29. C. Aguilar-Ibañez, O. O. Gutiérrez-Frias, M. S. Suarez-Castanon, Controlling the strongly damping inertia wheel pendulum via nested saturation function, Computación y Sistemas 12 (4) (2009) 436-449.

30. N. Qaiser, N. Iqbal, A. Hussain, N. Qaiser, Exponential stabilization of the inertia wheel pendulum using dynamic surface control, Journal of Circuits, Systems and Computers 16 (01) (2007) 81-92.

31. N. Touati, A. Chemori, Predictive control for the stabilization of a fast mechatronic system : From simulation to real-time experiments, in: Proceedings of the International IFAC Symposium on Mechatronic Systems, 2013, pp. 237-242.

32. M. Olivares, P. Albertos, On the linear control of underactuated systems: The flywheel inverted pendulum, in: Proceedings of the 10th IEEE International Conference on Control and Automation, 2013, pp. 27-32.

33. M. Olivares, P. Albertos, Linear control of the flywheel inverted pendulum, ISA Transactions 53 (5) (2014) 1396-1403.

34. N. H. Khraief, A. Chemori, S. Belghith, External disturbance rejection in IDA-PBC controller for underactuated mechanical systems: From theory to real time experiments, in: Proceedings of the IEEE Conference on Control Applications, 2014, pp. 1747-1752.

35. N. H. Khraief, A. Chemori, J. J. Pena, S. Belghith, Stabilization of inertia wheel inverted pendulum by model reference adaptive IDA-PBC: From simulation to real-time experiments, in: Proceedings of the 3rd International Conference onControl, Engineering Information Technology (CEIT), 2015, pp. 1-6.

36. M. Ryalat, D. S. Laila, A simplified IDA-PBC design for underactuated mechanical systems with applications, European Journal of Control 27 (2016) 1-16.

37. Z.-Q. Guo, J.-X. Xu, T. H. Lee, Design and implementation of a new sliding mode controller on an underactuated wheeled inverted pendulum, Journal of the Franklin Institute 351 (2014) 2261-2282.

38. S. Mobayen, Design of LMI-based sliding mode controller with an exponential policy for a class of underactuated systems, Complexity (2014) 1-8.

39. M. Yue, X. Sun, N. Li, C. An, Dynamic motion planning and adaptive tracking control for a class of two-wheeled autonomous vehicle with an underactuated pendular suspension, Journal of Dynamic Systems, Measurement, and Control 137 (10) (2015) DS-15-1019.

40. A. Zhang, C. Yang, S. Gong, J. Qiu, Nonlinear stabilizing control of underactuated inertia wheel pendulum based on coordinate transformation and time-reverse strategy, Nonlinear Dynamics 84 (4) (2016) 2467-2476.

41. L. T. Aguilar, I. Boiko, L. Fridman, R. Iriarte, Generating self-excited oscillations via two-relay controller, IEEE Transaction on Automatic Control 54 (2) (2009) 416-420.

42. L. T. Aguilar, I. Boiko, L. Fridman, R. Iriarte, Generating self-excited oscillations for underactuated mechanical systems via two-relay controller, International Journal of Control 82 (9) (2009) 1678-1691.

43. L. T. Aguilar, I. Boiko, L. Fridman, L. Freidovich, Generating oscillations in inertia wheel pendulum via two relay controller, International Journal of Robust and Nonlinear Control 22 (2012) 318-330.

44. R. Iriarte, L. T. Aguilar, L. Fridman, Second order sliding mode tracking controller for inertia wheel pendulum, Journal of the Franklin Institute 350 (2013) 92-106.

45. L. T. Aguilar, I. Boiko, L. Fridman, R. Iriarte, Self-Oscillations in Dynamic Systems: A New Methodology via Two-Relay Controllers, 1st Edition, Birkhauser Basel, Springer International Publishing Switzerland, 2015.

46. A. Estrada, L. T. Aguilar, R. Iriarte, L. Fridman, Two relay controller for real time trajectory generation and its application to inverted orbital stabilization of inertia wheel pendulum via quasi-continuous HOSM, Asian Journal of Control 14 (1) (2012) 58-66.

47. L. B. Freidovich, P. X. L. Hera, U. Mettin, A. Robertsson, A. S. Shiriaev, R. Johansson, Shaping stable periodic motions of inertia wheel pendulum: Theory and experiment, Asian Journal of Control 11 (5).

48. A. S. Shiriaev, J. W. Perram, C. C. de Wit, Constructive tool for orbital stabilization of underactuated nonlinear systems: Virtual constraints approach, IEEE Transaction on Automatic Control 50 (2005) 1164-1176.

49. A. Shiriaev, J. Perram, A. Robertsson, A. Sandberg, Periodic motion planning for virtually constrained Euler-Lagrange systems, Systems and Control Letters 55 (2006) 900-907. 
50. A. S. Shiriaev, L. B. Freidovich, I. R. Manchester, Can we make a robot ballerina perform a pirouette? orbital stabilization of periodic motions of underactuated mechanical systems, Annual Reviews in Control 32 (2) (2008) 200-211.

51. C. Gruber, M. Hofbaur, Periodic Motion Control of the Reaction Wheel Pendulum, in: 13th Mechatronic Forum, 2012, pp. 1-8.

52. S. Andary, A. Chemori, S. Krut, Stable Limit Cycle Generation for Underactuated Mechanical Systems, Application: Inertia Wheel Inverted Pendulum, in: Proceedings of the IEEE/RSJ International Conference on Intelligent RObots and Systems, 2008, pp. 526-531.

53. S. Andary, A. Chemori, S. Krut, Control of the underactuated inertia wheel inverted pendulum for stable limit cycle generation, Advanced Robotics 23 (15) (2009) 1999-2014.

54. S. Andary, A. Chemori, S. Krut, Estimation-based disturbance rejection in control for limit cycle generation on inertia wheel inverted pendulum testbed, in: Proceedings of the IEEE/RSJ International Conference on Intelligent RObots and Systems, 2009, pp. 1302-1307.

55. C. Zayane-Aissa, T.-M. Laleg-Kirati, A. Chemori, Control of a perturbed under-actuated mechanical system, in: Proceedings of the IEEE Multi-Conference on Systems and Control, 2015, pp. 294-299.

56. C. A.-I. anez, J. C. Martinez, J. de Jesus Rubio, M. S. Suarez-Castanon, Inducing sustained oscillations in feedback-linearizable single-input nonlinear systems, ISA Transactions 54 (2015) 117-124.

57. D. M. Alonso, E. E. Paolini, J. L. Moiola, Global bifurcation analysis of a controlled underactuated mechanical system, Nonlinear Dynamics 40 (3) (2005) 205-225.

58. D. M. Alonso, E. E. Paolini, J. L. Moiola, Controlling an inverted pendulum with bounded controls, in: F. Colonius, L. Grüne (Eds.), Dynamics, Bifurcations, and Control, Vol. 273 of Lecture Notes in Control and Information Sciences, Springer Berlin Heidelberg, 2002, pp. 3-16.

59. D. Pagano, L. Pizarro, J. Aracil, Local bifurcation analysis in the Furuta pendulum via normal forms, International Journal of Bifurcation and Chaos 10 (5) (2000) 981-995.

60. S. Nikolov, V. Nedev, Bifurcation analysis and dynamic behaviour of an inverted pendulum with bounded control, Journal of Theoretical and Applied Mechanics 64 (1) (2016) 17-32.

61. S. Boyd, L. El-Ghaoui, E. Feron, V. Balakrishnan, Linear matrix inequalities in system and control theory, 1st Edition, Vol. 15 of Studies in Applied and Numerical Mathematics, SIAM, Philadelphia, PA., 1994.

62. I. R. Petersen, R. Tempo, Robust control of uncertain systems: Classical results and recent developments, Automatica 50 (5) (2014) 1315-1335.

63. R. C. L. F. Oliveira, M. C. de Oliveira, P. L. D. Peres, Robust state feedback LMI methods for continuous-time linear systems: Discussions, extensions and numerical comparisons, in: 2011 IEEE International Symposium on Computer-Aided Control System Design (CACSD), 2011, pp. 1038-1043.

64. L. A. Rodrigues, R. C. L. F. Oliveira, J. F. Camino, New extended LMI characterization for state feedback control of continuous-time uncertain linear systems, in: Control Conference (ECC), 2015 European, 2015, pp. 1992-1997.

65. F. Pfeiffer, C. Glocker, Multibody Dynamics with Unilateral Contacts, Wiley Series in Nonlinear Science, Wiley-VCH Verlag GmbH \& Co. KGaA, Weinheim, 2004.

66. A.-C. Huang, Y.-C. Chen, Adaptive sliding control for single-link flexible-joint robot with mismatched uncertainties, IEEE Transactions on Control Systems Technology 12 (5) (2004) 770-775.

67. M. Hosseinpour, P. Nikdel, M. Badamchizadeh, M. Akbari, Modelling and control of flexible joint robot based on Takagi-Sugeno fuzzy approach and its stability analysis via sum of squares, Mathematical and Computer Modelling of Dynamical Systems 19 (3) (2013) 250-262.

68. K. Merat, H. Salarieh, A. Alasty, A. Meghdari, Stochastic piecewise affine control with application to pitch control of helicopter, Nonlinear Analysis: Hybrid Systems 15 (2015) 86-97.

69. H. Razavi, K. Merat, H. Salarieh, A. Alasty, A. Meghdari, Observer based minimum variance control of uncertain piecewise affine systems subject to additive noise, Nonlinear Analysis: Hybrid Systems 19 (2016) 153-167.

70. R. C. Luo, J. Sheng, C. C. Chen, P. H. Chang, C. I. Lin, Biped robot push and recovery using flywheel model based walking perturbation counteraction, in: 13th IEEE-RAS International Conference on Humanoid Robots (Humanoids), 2013, pp. 50-55.

71. R. C. Luo, C. W. Huang, A push-recovery method for walking biped robot based on 3-D flywheel model, in: 41st Annual Conference of the IEEE Industrial Electronics Society, IECON 2015, 2015, pp. $2685-2690$.

72. M. Shafiee-Ashtiani, A. Yousefi-Koma, M. S. Panahi, M. Khadiv, Push recovery of a humanoid robot based on model predictive control and capture point, CoRR abs/1612.08034.

URL http://arxiv.org/abs/1612.08034 
73. J. Pratt, J. Carff, S. Drakunov, A. Goswami, Capture point: A step toward humanoid push recovery, in: 2006 6th IEEE-RAS International Conference on Humanoid Robots, 2006, pp. 200-207.

74. E. R. Westervelt, J. W. Grizzle, C. Chevallereau, J.-H. Choi, B. Morris, Feedback control of dynamic bipedal robot locomotion, Taylor \& Francis/CRC, London, 2007.

75. C. E. de Brito Novaes, P. S. P. da Silva, P. Rouchon, Trajectory control of a bipedal walking robot with inertial disc, IFAC Proceedings Volumes 47 (3) (2014) 4843-4848, 19th IFAC World Congress.

76. C. E. de Brito Novaes, P. S. P. da Silva, Numerical estimation of stability region of self-clocked bipedal robots with inertial disk, Journal of Control, Automation and Electrical Systems 27 (6) (2016) 634-645.

77. B. M. Fard, A. Bagheri, N. Nariman-Zadeh, Limit cycle walker push recovery based on a receding horizon control scheme, Proceedings of the Institution of Mechanical Engineers, Part I: Journal of Systems and Control Engineering 226 (7) (2012) 914-926.

78. Y. Zhu, Y. Gao, C. Xu, J. Zhao, H. Jin, J. Lee, Adaptive control of a gyroscopically stabilized pendulum and its application to a single-wheel pendulum robot, IEEE/ASME Transactions on Mechatronics 20 (5) (2015) 2095-2106.

79. M. W. Spong, M. Vidyasagar, Robot Dynamics and Control, 1st Edition, John Wiley \& Sons, New York, NY, USA, 1989.

80. T. S. Parker, L. O. Chua, Practical numerical algorithms for chaotic systems, Springer, New York, 1989.

81. C. Scherer, S. Weiland, Linear Matrix Inequalities in Control, Dutch Institute of Systems and Control (DISC), The Netherlands, 2005.

82. M. M. Asheghan, M. T. Beheshti, An LMI approach to robust synchronization of a class of chaotic systems with gain variations, Chaos, Solitons \& Fractals 42 (2) (2009) 1106-1111.

83. S. Mobayen, An LMI-based robust controller design using global nonlinear sliding surfaces and application to chaotic systems, Nonlinear Dynamics 79 (2) (2015) 1075-1084.

84. T. Kailath, Linear Systems, Prentice-Hall, Englewood Cliffs, NJ, 1989.

85. N. J. Higham, Accuracy and Stability of Numerical Algorithms, 2nd Edition, Society for Industrial and Applied Mathematics, Philadelphia, PA, USA, 2002.

86. J. G. VanAntwerp, R. D. Braatz, A tutorial on linear and bilinear matrix inequalities, Journal of Process Control 10 (4) (2000) 363-385.

87. P. Gahinet, A. Nemirovski, A. J. Laub, M. Chilali, LMI control toolbox. For Use with MATLAB, MathWorks, Natick, Massachusetts, 1995.

88. H. Gritli, S. Belghith, Walking dynamics of the passive compass-gait model under OGY-based control: Emergence of bifurcations and chaos, Communications in Nonlinear Science and Numerical Simulation 47 (2017) 308-327.

89. H. Gritli, S. Belghith, N. Khraeif, OGY-based control of chaos in semi-passive dynamic walking of a torso-driven biped robot, Nonlinear Dynamics 79 (2) (2015) 1363-1384.

90. H. Gritli, S. Belghith, Bifurcations and chaos in the semi-passive bipedal dynamic walking model under a modified OGY-based control approach, Nonlinear Dynamics 83 (4) (2016) 1955-1973.

91. Z. Wang, L. Zhou, Z. Chen, J. Wang, Local bifurcation analysis and topological horseshoe of a 4D hyper-chaotic system, Nonlinear Dynamics 83 (4) (2016) 2055-2066.

92. L. Zhou, Z. Chen, Z. Wang, J. Wang, On the analysis of local bifurcation and topological horseshoe of a new 4D hyper-chaotic system, Chaos, Solitons \& Fractals 91 (2016) 148-156.

93. W. Wu, Z. Chen, Hopf bifurcation and intermittent transition to hyperchaos in $\hat{A}$ aÂ novel strong four-dimensional hyperchaotic system, Nonlinear Dynamics 60 (4) (2010) 615-630.

94. X. Li, P. Wang, Hopf bifurcation and heteroclinic orbit in a 3D autonomous chaotic system, Nonlinear Dynamics 73 (1) (2013) 621-632.

95. A. Algaba, M. C. Domínguez-Moreno, M. Merino, A. J. Rodríguez-Luis, Study of the Hopf bifurcation in the Lorenz, Chen and Lü systems, Nonlinear Dynamics 79 (2) (2015) 885-902.

96. K. Deng, S. Yu, Hopf bifurcation analysis of a new modified hyperchaotic Lü system, Optik - International Journal for Light and Electron Optics 124 (23) (2013) 6265-6269.

97. W. Govaerts, Numerical bifurcation analysis for ODEs, Journal of Computational and Applied Mathematics $125(1-2)(2000) 57-68$.

98. A. Dhooge, W. Govaerts, Y. A. Kuznetsov, MATCONT: A MATLAB package for numerical bifurcation analysis of ODEs, ACM Transactions on Mathematical Software 29 (2) (2003) 141-164. 